\title{
Especial
}

\section{Prevención del suicidio en médicos}

\section{Suicide prevention in doctors}

\section{José Carlos Mingote Adán ', Dolores Crespo Hervás ${ }^{2}$, Matilde Hernández Álvarez ${ }^{3}$, Mercedes Navío ${ }^{4}$, Consuelo Rodrigo García-Pando 5}
1. Psiquiatra Coordinador del Programa de Atención Integral al Personal Sanitario Enfermo de la Comunidad Autónoma de Madrid.
2. Presidenta del Programa de Atención Integral al Médico Enfermo del llustre Colegio de Médicos de Madrid.
3. Universidad Autónoma de Madrid/Clínica Tavistock de Londres. Responsable de los Programas de Formación sobre Detección y Prevención de la Depresión y el Suicidio para Médicos de Atención Primaria y Profesionales que trabajan con Pacientes Geriátricos de la Comunidad Autónoma de Madrid.
4. Directora Asistencial de la oficina Regional de Salud Mental de la Comunidad Autónoma de Madrid.
5. Servicio de Prevención de Riesgos Laborales del Hospital Universitario Severo Ochoa.

\section{Recibido: 12-03-13}

Aceptado: 21-05-13

\section{Correspondencia}

José Carlos Mingote Adán.

Unidad de Coordinación del Programa de Atención Integral al Personal Sanitario Enfermo de la Comunidad Autónoma de Madrid.

PAITSE. Madrid. España.

Tfno. 913303926

josecarlos.mingote@salud.madrid.org

\section{Resumen}

Se sabe que la tasa de suicidio en médicos es mayor que en la población general. La salud de los médicos ha recibido escasa atención de sus colegas, a pesar de la considerable y creciente evidencia de que la morbilidad es alta en la profesión. Cuidar de otras personas genera estrés en los cuidadores. El estrés por la incertidumbre clínica y el temor al fracaso son rasgos importantes de la cultura médica. La paradoja es que el cuidado de los pacientes genera estrés pero es también la fuente de gratificación más importante del médico. Se sabe que aquellos que cuidan de los demás a menudo tienen grandes dificultades para buscar y recibir ayuda para ellos mismos. Las tasas de suicidio de los médicos en Estados Unidos, Inglaterra y otros países son más altas que las de la población general y las de otras profesiones. La tasa de suicidio de los médicos es ligeramente mayor que la de la población general, mientras que la de las médicas es claramente superior. Esta tendencia es más pronunciada entre psiquiatras y anestesistas. Los médicos tienen unas necesidades asistenciales específicas por sufrir elevados niveles de alcoholismo, dependencia de drogas, rupturas matrimoniales, enfermedad mental y suicidio. La automedicación es muy frecuente entre los médicos, en especial con hipnóticos, antidepresivos y analgésicos opiáceos. Muchas de estas dificultades están relacionadas con el estrés, elevada responsabilidad y tiempo insuficiente para trabajar y vivir conciliando las demandas laborales y familiares. Muchos médicos tienen grandes dificultades para discutir sus problemas con los colegas y se tratan a ellos mismos, de forma notablemente peligrosa. El fácil acceso de los médicos a las drogas implica que el abuso de drogas sea un riesgo ocupacional potencial de hombres y mujeres.

Med Segur Trab (Internet) 2013; 59 (231) 176-204

Palabras clave: La salud de los médicos, organización del trabajo, médicos, depresión, adicciones, riesgo de suicidio 


\section{Abstract}

The rate of suicide in physicians is known to be higher than that of the general public. The health of doctors has always received scant attention from their colleagues, in spite of considerable and increasing evidence that morbidity and mortality are high in the profession. Caring for others imposes considerable strains on the careers. The stress by clinical uncertainty and the fear of failure are important parts of the medical ethos. It is recognized that those who do care for others often have great difficulty in asking for, and receiving, care for themselves. Suicide rates for doctors in the United States, United Kingdom and other countries are higher than general population and other professions. The suicide rate of male physicians is slightly higher than that of the general population, while that of their female colleagues is clearly higher. This tendency is most pronounced in female psychiatrist and anesthetists. Doctors have specific needs for health care by suffer abnormally high levels of alcoholism, drug dependence, marital breakdown, mental illness and suicide. The self-medication is common among doctors, particularly sleeping tablets, antidepressants and opiate analgesics. Many of the difficulties are related to stress, high responsibility and insufficient time to do the work and to live reconciling demands between work and family. Many doctors show an increased impediment for discuss their problems with colleagues, and treat themselves, which is notoriously hazardous. Doctors' easy access to drug makes drug abuse a potential occupational hazard for men and women.

Med Segur Trab (Internet) 2013; 59 (231) 176-204

Key words: The bealth of doctors, work organization, physicians, depression, addictions, suicide risk. 


\section{INTRODUCCIÓN}

¿Es posible cumplir esta recomendación de un médico ateniense 2 a. C? : "Estos son los deberes de un médico: Primero... sanar su mente y ayudarse a sí mismo antes de ayudar a nadie”: ¿Pero cómo?

"Existe la creencia (errónea) de que los médicos lo tienen todo: una profesión, un trabajo estimulante, prestigio, inteligencia, éxito, y sobre todo dinero y apariencias" (Myers 2006).

"Tradicionalmente, los suicidios de los médicos son ocultados, negados, motivo de cotilleo, pero no abiertamente tratados de forma colectiva por los colegas. Hoy, con más conocimiento y menos estigma, las comunidades médicas están pudiendo afrontar las muertes trágicas de sus compañeros" (Myers y Gabbard 2008).

"Como médico y como político, estoy muy preocupado por la cuestión de cómo las enfermedades pueden afectar a la toma de decisiones de los jefes de estado o de gobierno... Propongo que en una democracia deberíamos tomar las medidas necesarias para asegurar que antes de votar a un candidato para la Jefatura del Estado o como Primer Ministro, el electorado debería conocer los resultados de un examen médico actualizado e independiente..., así como a intervalos regulares, teniendo en cuenta las complejidades de la dinámica del gobierno, y las relaciones inciertas entre enfermedad y la capacidad para tomar decisiones" (Owen 2003). De igual modo, es necesario evaluar la salud mental de los profesionales de empleos que requieren una elevada fiabilidad, porque de ellos depende la seguridad de la población, así como tener en cuenta las condiciones en las que realizan su actividad laboral, para que sean las más convenientes para el ejercicio de su actividad profesional y controlar posibles riesgos psicosociales que puedan dificultar su importante misión social y dañar su salud personal.

Si el método científico es un modelo de predicción del comportamiento de la naturaleza, la medicina ha sido considerada como "la ciencia y el arte de manejar la incertidumbre" (Neighbour, 1998). Para lograrlo, el médico precisa de habilidades complejas como las que se requieren para la entrevista clínica, estar en contacto y saber utilizar las propias emociones ("la consulta interior"), técnicas de comunicación y aprender a cuidar de sí mismo para poder cuidar también de los demás. Mantener de forma continuada la competencia profesional exige del médico el aprendizaje de nuevos conocimientos y habilidades, tanto de competencias científico-técnicas como psicosociales. Implica la posibilidad de establecer relaciones éticamente adecuadas con los pacientes, en las que se respete el principio de confidencialidad, la búsqueda de la adherencia terapéutica, las responsabilidades profesionales y la calidad asistencial. Estas responsabilidades requieren que los médicos cuenten con los adecuados recursos de autorregulación eficaz, individual y colectiva, así como para la identificación, notificación y ayuda a los médicos que no cumplan los estándares de competencia profesional por motivos relacionados con su salud mental. El médico puede llegar a estar incapacitado para el cuidado de sí mismo y para el desarrollo de sus funciones asistenciales debido a la existencia de un trastorno mental grave y/o adictivo por la afección de sus capacidades cognitivas, afectivas y conductuales.

\section{PRIMEROS ESTUDIOS}

"Felipe Trigo (1865-1916), médico y escritor, en su novela El médico rural (1912), describe vivencias posiblemente autobiográficas, sobre la ansiedad que vive el joven médico rural por la escasa preparación profesional, la desesperanza e impotencia ante la muerte del enfermo y la aparición de la ideación suicida como solución. En 1916, Trigo se disparó un tiro en la sien" (de la Cruz, Corominas y Sarró 1988).

Ya en 1947 Dublin y Spiegelman estudian la longevidad y las causas de muerte de una serie de médicos en Estados Unidos y concluyen que:"es notable descubrir que los 
hombres y las mujeres de la profesión, dedicados a mejorar la salud de los demás, fracasan en proteger la de ellos mismos". Destacan una mayor tasa de mortalidad cardiovascular prematura en los médicos (un $80 \%$ mayor que población general), concluyendo que la enfermedad coronaria era un riesgo ocupacional de estos profesionales.

En 1955 Dowling (citado por King) describió el conocido triple signo de "ignorancia, indiferencia y falta de cuidado" de la propia salud por los médicos. King (1970) estudia las causas de mortalidad en varones de cuatro profesiones (abogados, maestros, médicos y sacerdotes) en varios países europeos y en Norteamérica, y destaca la mayor morbimortalidad por enfermedad coronaria, cerebrovascular y por suicidio en médicos. Este mismo autor propone un modelo teórico, según el cual el nivel de salud de los miembros de una profesión depende de la interacción de tres tipos de factores: los antecedentes individuales, el medio laboral y los hábitos de conducta de salud/enfermedad, como la dieta, el consumo etílico y el ejercicio físico.

Desde el editorial en el British Medical Journal de 1964, el tópico del suicidio del médico ha sido objeto de numerosos estudios, aunque una cuidadosa revisión de los mismos muestra en ellos varios problemas metodológicos, como incluir muestras pequeñas, breve periodo de tiempo para la recogida de datos, inadecuados controles para edad y sexo, e insuficiente estandarización de los criterios para la muestra y los grupos de referencia.

Craig y Pitts (1968) revisan las tasas de suicidio por especialidades y sugieren que los psiquiatras tienen una tasa más alta que los médicos de otras especialidades, aunque las muestras son tan pequeñas que no permiten sacar conclusiones definitivas. También, Blachly, Disher y Roduner (1968) estiman las tasas de suicidio para las diferentes especialidades, que varían desde 61 por 100.000 para los psiquiatras hasta 10 por 100.000 para los pediatras, igual que en la población general. El momento temporal del suicidio es diferente entre los médicos, que se eleva hacia la edad media de la vida (entre 45 y 64 años) según revisan Steppacher y Mausner (1974). En esta revisión se constata que las médicas, especialmente las más jóvenes tienen un riesgo tres veces mayor que las mujeres en general, hasta igualar las tasas de suicidio de sus colegas varones.

Craig y Pitts (1968) comunicaron una tasa 4 veces mayor que la de mujeres de la población general, atribuida a una elevada prevalencia de trastornos depresivos. Sin embargo en un estudio nacional Rich y Pitts (1979) examinaron 544 médicos suicidas y controles ajustados por edad del periodo 1967-1972 y concluyeron que no existen diferencias entre la tasa de suicidio de médicos varones y controles adecuados en Estados Unidos. Su tasa de 35,7 suicidios por 100.000 médicos no difiere de la de los varones de la población general, que era de 34,6 por 100.000 varones blancos. Según este estudio, los psiquiatras se suicidan el doble de lo esperado. También, Blachly, Disher y Roduner (1968) estiman las tasas de suicidio para las diferentes especialidades, que varían desde 61 por 100.000 para los psiquiatras hasta 10 por 100.000 para los pediatras, igual que en la población general. El momento temporal del suicidio es diferente entre los médicos, y se eleva hacia la edad media de la vida (entre 45 y 64 años) según revisan Steppacher y Mausner (1974). En esta revisión se constata que las médicas, especialmente las jóvenes tienen al menos un riesgo tres veces mayor que las mujeres en general, hasta igualar las tasas de suicidio de sus colegas varones. El método de suicidio más frecuentemente empleado, el envenenamiento, ha sido consistentemente comunicado en todos los estudios.

La subestimación general de las tasas de suicidio ha sido apoyada por los resultados del estudio de Rose and Rosow (1973) realizado sobre una base de 48 suicidios de médicos en California durante un periodo de 3 años. Concluyó que entre los médicos, los psiquiatras tienen las tasas más elevadas de suicidio. Se cree que la tasa de suicidio médico es realmente mayor que el registrado porque con frecuencia puede ser mal codificado deliberadamente en los certificados de muerte como sobredosis accidental, debido al elevado nivel de estigma. 
Según los resultados del estudio sobre las causas de muerte entre los médicos ingleses (Registrar General 1978), estos tienen más riesgo de morir por suicidio, cirrosis hepática (un riesgo 3 veces mayor) y accidentes de tráfico (casi el doble), en comparación con población general. El alcoholismo es el trastorno psiquiátrico que más frecuentemente perjudica el cuidado de sus pacientes y les involucra en procedimientos disciplinarios.

Preven (1983) realiza una revisión sistemática de los estudios realizados sobre el suicidio de los médicos y concluye que el suicidio en médicos varones tiene lugar en la misma tasa que sus controles ajustados por edad en Estados Unidos. Igualmente, este mismo autor revisa la cuestión del suicidio de las mujeres médicos y concluye que 3 estudios confirman que la tasa de suicidio de las médicas es 3-4 veces superior a la de los controles no médicos, y con una tasa similar a la de sus compañeros varones. Los psiquiatras parecen estar sobre representados en el porcentaje de médicos que se suicidan. (Preven 1983).

Ducker (1987) revisa los estudios realizados y concluye, a pesar de la controversia existente, que se puede explicar por una elevada tasa de trastornos afectivos no adecuadamente tratados en las médicas.

La revisión bibliográfica de de la Cruz, Corominas y Sarró (1988) concluye que: Los profesionales de la medicina presentan una prevalencia de suicidio superior a la de la población general. El método más usado por los médicos es el empleo de medicamentos que ellos mismos pueden proporcionarse. Los especialistas con elevado riesgo suicida son los psiquiatras, los otorrinolaringólogos, cirujanos, anestesistas y dentistas.

Al revisar el tratamiento estadístico de los datos de estos primeros estudios se apreciaron varias debilidades metodológicas, como la presencia de una inadecuada estandarización, el limitado tamaño muestral y la ausencia de grupo control adecuado que limitan su validez, aunque han tenido el mérito de llamar la atención sobre este problema.

\section{ESTUDIOS POSTERIORES}

Posteriormente se han realizado estudios epidemiológicos metodológicamente más válidos, con muestras más amplias, como el de Arnetz et al (1987) siguieron una cohorte nacional de médicos, profesores universitarios y un grupo control por un periodo de 10 años, habiendo identificado todos los casos de suicidio durante el periodo 1961-1970. Los resultados muestran una elevada tasa estandarizada de mortalidad (TEM) por suicidio entre médicas, comparadas con profesoras universitarias y población general. Los médicos muestran una elevada tasa de suicidio en comparación sólo con los profesores de universidad, y sobre todo por cirujanos generales respecto de otros especialistas.

Una revisión sistemática de 14 estudios internacionales en médicos, en artículos publicados desde 1963 hasta 1991, encontró mayores tasas de suicidio en médicos comparadas con las de la población general. El riesgo relativo osciló entre 1,1 y 3,4 en varones y de 2,5 a 5,7 en médicas (Lindeman et al 1996) estos mismos autores revisaron las historias clínicas de médicos, ingenieros y profesores finlandeses que se suicidaron entre 1986 y 1993. Los médicos tienen más diagnósticos somáticos que los grupos de referencia, y la prevalencia del diagnóstico de depresión fue mayor entre las médicas que entre los médicos, como en población general. La depresión fue la causa contributaria de muerte más prevalente en todos los grupos estudiados, aunque la mayoría de los médicos varones no recibían tratamiento. Igualmente revisó las tasas estandarizadas de mortalidad para diferenciar las de varones y mujeres. Concluyó que las médicas presentan un mayor riesgo de suicidio en comparación con la población general y otras mujeres profesionales (Lindeman et al 1997).

Otro gran estudio realizado en Inglaterra y Gales (1979-1995) confirmó la existencia de unas elevadas tasas de suicidio en médicas pero no en médicos (Hawton et al 2001). 
En 1999 Frank y Dingle estudiaron la prevalencia-vida de depresión auto identificada y los intentos de suicidio en la muestra del Estudio de Salud de las Médicos mujeres $(\mathrm{N}=4,501)$ y estimaron que $1,5 \%(\mathrm{~N}=61)$ de las médicas habían realizado un intento de suicidio, y un 19,5\% ( $\mathrm{N}=808)$ tenían antecedentes personales de depresión u otro trastorno psiquiátrico, lo que se asociaba con insatisfacción profesional, tener poco control sobre el trabajo y padecer un elevado estrés laboral, de forma que mayores niveles de depresión se asocian a un mayor riesgo de realizar intentos de suicidio. La depresión es aproximadamente tan común entre mujeres médicos como entre otras mujeres de Estados Unidos, aunque los intentos de suicidio pueden ser menores. Este mismo grupo realizó un gran estudio de de mortalidad proporcional en médicos blancos en 28 estados americanos desde 1984 hasta 1995 y encontraron que, comparados con otros profesionales, su tasa de mortalidad por suicidio fue mayor que por todas las demás causas de muerte. En población general la tasa de suicidio de los varones es 4 veces mayor que la de las mujeres, mientras que entre las médicas es tan alta como la de los médicos (Silverman2000). Una tasa tan alta de suicidios consumados puede resultar del mayor conocimiento de los médicos de toxicología y de acceso a drogas letales, ya que la sobredosis de medicamentos es el principal método de suicidio.

Aasland et al (2001) estudió las tasas de suicidio de los médicos noruegos entre 1960 y 1989 en comparación con otros trabajadores con y sin educación universitaria como controles. Se detectaron 82 suicidios en médicos, de los que 9 son mujeres, 265 personas suicidadas por personas con formación universitaria, y 11.165 por personas sin formación universitaria. La tasa de suicidio es medida por el número de muertes por 100.000 personas año. Las tasas de suicidio son 47,7 (95\% CI 37,7-60,4) por médicos varones, 20,1 $(17,7-22,9)$ por otros universitarios, y $22,7(22,2-23,2)$ para varones no universitarios. Las tasas de suicidio para las mujeres son: $32,3(15,8-63,7), 13,0(8,4-19,8)$ y $7,7(7,5-8,0)$. Las tasas de suicidio de los médicos son significativamente mayores que las de otros universitarios. El riesgo de suicidio de los médicos varones aumentó a medida que aumentaba su edad, sobre todo si permanecía soltero, en una medida 5 veces mayor que sus colegas casados. Para el $52 \%$ de los médicos y el $85 \%$ de las médicas el método de suicidio fue por envenenamiento, en una medida doble que en la proporción general.

Como la ideación suicida es un significativo factor de riesgo de intentos de suicidio y de suicidio consumado, Hem et al (2000) estudiaron la presencia de ideas de suicidio en una encuesta anónima de una muestra representativa de médicos noruegos $(\mathrm{N}=1063$ de un total de 1.476 médicos noruegos), de los que el 51,1\% reconocieron sentimientos y pensamientos de que la vida no merece la pena ser vivida y 1,6\% habían realizado algún intento de suicidio. Los factores de riesgo eran ser mujer, vivir sólo y padecer sintomatología depresiva no tratada de modo eficaz. Los pensamientos de suicidio eran rigurosamente atribuidos a sus condiciones laborales, aunque la sintomatología depresiva es la que se asocia fuertemente con la ideación autolítica. Una alta tasa de suicidio y una baja tasa de intentos de suicidio apoyan la hipótesis de que los médicos no piden ayuda, sino que tienden a actuar sus impulsos suicidas. Este mismo grupo comparó las tasas de suicidio según el nivel educativo en Noruega entre los años 1960 y 2000, con inclusión de 46 y 49 millones de personas-años entre hombres y mujeres respectivamente. Los médicos tienen una mayor tasa en comparación con otros graduados y con la población general, tanto entre varones (43.0, 95\% intervalo de confianza (CI) 35.3-52.5) y entre mujeres $(26.1,95 \%$ CI 15.1-44.9). Las tasas de suicidio aumentaron de forma aguda según se incrementaba la edad entre médicos y otros graduados universitarios, en comparación con los no universitarios.

Posteriormente otros estudios han constatado una elevada prevalencia de ideas de suicidio en médicos, así Gyorffy et al (2005) estudian una muestra de médicos húngaros ( $\mathrm{N}=407,298$ mujeres y 109 varones) y un grupo control de 1754 profesionales de igual nivel. Resultó que un $20,3 \%$ de médicas y un $12,15 \%$ de médicos tienen reconocen tener ideación autolítica, en mayor medida que el grupo control (12,3\% y 7,6\% respectivamente). Lasa médicas tienen una prevalencia significativamente mayor de ideas de suicidio $(p=0,0058, O R=1,901)$. Las ideas de suicidio se asociaban con estrés laboral relacionado 
con: jornadas muy prolongadas de trabajo ( $>8$ horas), ansiedad grave, estrés y conflicto de rol. Igualmente Van der Heijden et al (2008) estudian la prevalencia de burnout y de ideas de suicidio en una muestra representativa de residentes alemanes $(\mathrm{N}=2115)$. Resulta que el 20,6\% son clasificados como con burnout, el $12 \%$ reconocen haber tenido ideas de suicidio alguna vez durante la residencia y un $1 \%$ muchas veces. Los pensamientos de suicidio son más prevalentes en los residentes con burnout en comparación con los que no lo tienen $(20,5 \%$ versus $7,6 \%, \mathrm{p}<.001)$. El conocimiento de esta relación entre burnout e ideas de suicidio puede ser importante para la prevención del suicidio.

Según la Fundación Americana para la Prevención del Suicidio (2002), los estudios realizados han confirmado que los médicos mueren por suicidio más frecuentemente que otras personas de su misma edad y género de la población general y de otras profesiones. De media, la muerte por suicidio es $70 \%$ más frecuente entre médicos varones que en otros profesionales de los Estados Unidos, y 250\%-400\% mayor entre las médicas. A diferencia de otras poblaciones, en los que los hombres se suicidan cuatro veces más a menudo que las mujeres, los médicos tienen una tasa de suicidio que es muy parecida entre hombres y mujeres. Esta Fundación constituyó un grupo de trabajo de 15 expertos para evaluar el estado del conocimiento sobre la depresión y el suicidio en los médicos, y las barreras que les impide tratarse. Tras la jornada de trabajo concluyeron en el documento de consenso, que la cultura tradicional de la medicina otorga escasa prioridad al cuidado de la salud mental del médico a pesar de la evidencia de que presentan una elevada prevalencia de trastornos del estado de ánimo no adecuadamente tratados. Las barreras para que los médicos busquen ayuda son el temor a daños punitivos como la retirada de la licencia para ejercer la medicina, la pérdida de privilegios y su progreso profesional. Este grupo recomendó cambiar las actitudes profesionales y cambiar las políticas institucionales para animar a los médicos a pedir ayuda cuando lo necesiten y a ayudar a sus compañeros a reconocer y tratarse cuando lo precisen. Los médicos son tan vulnerables a la depresión como la población general, pero buscan ayuda en menor medida y las tasas de suicidio consumado son más altas (Center et al., 2003).

Schernhammer y Colditz (2004) realizaron un meta-análisis de 25 estudios de calidad sobre el suicidio médico y concluyeron que la tasa agregada de suicidio para los médicos varones comparada con la de los varones en la población general, es 1,41: 1, con un 95\% y un intervalo de confianza de 1,21 a 1,65. Para las médicas, la ratio fue 2,27: 1 (95\% $\mathrm{IC}=1,90-2,73$ ), en comparación con las mujeres en la población general; que es una tasa muy elevada.

En el estudio de Torre et al (2000) se comparan todas las causas de muerte en una muestra representativa de médicos de Estados Unidos con un grupo ajustado de población general desde 1948 hasta 1998. Resultó que el riesgo de muerte fue 56\% menor de lo esperado en los médicos y $26 \%$ menor en las médicas, en comparación con sus pares de la población general: La tasa de mortalidad estandarizada fue marcadamente menor para enfermedades asociadas al tabaquismo, como enfermedades cardiovasculares, respiratorias y cáncer de pulmón. El suicidio fue la única causa de muerte en la que el riesgo fue mayor que en población general.

Los estudios de los factores de riesgo de suicidio consumado suelen ser estudiados a través de la reconstrucción por medio de la autopsia psicológica, por entrevistas semiestructuradas a los informantes claves. En 1986 la Asociación Médica Americana realizó un estudio piloto sobre los médicos que habían consumado el suicidio y encontraron depresión y problemas por uso de sustancias no tratados, además de gran frecuencia de pérdidas personales y profesionales, problemas económicos, una tendencia a trabajar en exceso, elevado estrés e insatisfacción con la profesión. Un porcentaje significativo de médicos no tienen un médico personal y usan menos los servicios médicos preventivos. La cultura de la profesión no anima a los médicos a admitir sus vulnerabilidades ni a buscar la ayuda que necesitan, sobre todo en cuanto a sus problemas de salud mental. Las causas más citadas son la falta de tiempo (48\%), la falta de confidencialidad (37\%), el estigma (30\%), el coste (28\%), y el miedo a que conste en su expediente profesional (24\%) según la revisión de Center et al (2003).En varios de ellos se constata que ninguno de los 
médicos había sido diagnosticado ni tratado correctamente (Lindeman et al 1997). En el estudio de autopsia psicológica de Hawton, Malmberg y Simkin (2004), realizado en 38 médicos que habían muerto por suicidio en Inglaterra y Gales entre enero de 1991 y diciembre de 1993, se constató que el 65\% de ellos padecían un trastorno mental, sobre todo depresión y abuso de alcohol o drogas, por el que había consultado en menor medida que las personas de otros estudios análogos en población general. Veinticinco médicos habían comunicado tener importantes problemas laborales, y en menor proporción problemas de relación y económicos. El método más común de suicidio fue la auto-intoxicación con drogas que habían tomado de sus lugares de trabajo.

El proceso suicida ha sido empíricamente estudiado en el estudio prospectivo de una cohorte de estudiantes noruegos de medicina $(\mathrm{N}=631)$, primero al final de sus estudios de grado y posteriormente al final del primer y cuarto año de postgrado, con un tiempo medio de seguimiento de 3,6 años. El 6\% de ellos comunicaron tener planes de suicidio en los años de postgrado. Los predictores en el momento inicial son neuroticismo (rasgo de vulnerabilidad), síntomas depresivos graves y acontecimientos vitales estresantes negativos. Entre aquellos con ideas de suicidio en el momento inicial, el 8\% presentaron planificación suicida en los dos momentos de valoración posterior. Los predictores de transición desde pensamientos a planes de suicidio fueron el rasgo de juicio de realidad débil y la presencia de síntomas depresivos graves, aunque una minoría de los planificadores habían buscado ayuda profesional (Tyssen et al 2004).

Varios estudios (Shanafelt et al. 2011, Pompili et al. 2010) han constatado una elevada prevalencia de ideas de suicidio entre cirujanos americanos en los 12 meses previos (el 6,3\%), de 1,5 a 3 veces más común que en P.G. $(\mathrm{P}<.02)$, aunque sólo el $26 \%$ de ellos habían buscado ayuda psiquiátrica o psicológica, y el 60,1\% rechazan buscar ayuda por temor de que pudiese afectar su licencia médica. Tener idas recientes de suicidio se asocian significativamente con la presencia de elevados síntomas de depresión y burnout. Además, unos elevados niveles de burnout, sobre todo en agotamiento emocional y distanciamiento, según el Inventario de Burnout de Oldenburg, se asocian con elevados niveles de desesperanza en la escala de Desesperanza de Beck, un marcador psicométrico de riesgo de suicidio (Pompili et al. 2010).

Para determinar la existencia de diferencias de género en la incidencia de suicidio en médicos, y si existen diferencias en los métodos usados por ellos, Lagro-Janssen y Luijs revisaron en 2008 nueve estudios por reunir los criterios de calidad necesarios y concluyeron que el suicidio entre los médicos ocurrió en una tasa similar a la de la población general, mientras que el riesgo era superior en las médicas, siendo el uso de medicación el método más empleado. En otro estudio (Petersen y Burnett 2008) se revisan los certificados de defunción de médicos y dentistas frente a todos los trabajadores desde 1984 hasta 1992 en 26 estados norteamericanos. Se calcularon las TES según la edad, y resultó que la tasa de suicidio de las médicas era superior a la del grupo control (TES=2,39, 95\% CI=1,52-3,77). Para los médicos la TES era reducida $(0,80,95 \% \mathrm{CI}=0,53-1,20)$, salvo entre los de edad más avanzada que aumentaba de forma significativa.

Varios estudios epidemiológicos nacionales han constatado que los miembros de algunas ocupaciones están en mayor riesgo de suicidio que otras, y que la mayor parte de esta considerable variación en el riesgo de suicidio entre ocupaciones es explicable por factores socioeconómicos, salvo para médicos y enfermeras. Así en un estudio anillado caso-control con 3195 suicidios y 63900 controles emparejados en Dinamarca (Agerbo et al. 2007), se constató que comparados con maestros, la tasa de suicidio es 50\% mayor entre médicos, enfermeras, trabajadores no cualificados y en el grupo sin empleo. El riesgo de suicidio disminuye en todas las ocupaciones después de controlar las variables de: ingreso psiquiátrico, estatus de empleo, estatus marital e ingresos brutos, con la excepción de médicos y enfermeras, en los que la tasa de suicidio aumentó de nuevo. Entre las personas que han recibido tratamiento psiquiátrico hospitalario, se aprecian unas modestas asociaciones entre suicidio y ocupación, excepto para los médicos, que tienen un riesgo cuatro veces mayor. En gran parte, el mayor riesgo de suicidio de algunos grupos ocupacionales, puede reflejar un elevado estrés laboral y el acceso a medios 
letales de suicidio como medicinas o armas de fuego. Entre los médicos, el riesgo es mayor para los anestesistas por tener acceso a fármacos anestésicos empleados en muchas muertes por suicidio. Otros grupos de alto riesgo (como dentistas, farmacéuticos, veterinarios y granjeros) también tienen más fácil acceso a medios (Hawton, K. 2009). Aparte de los médicos, el riesgo de suicidio tiene poca asociación con la ocupación entre las personas que sufren un trastorno mental.

Desafortunadamente hay pocos estudios sobre trastornos mentales comórbidos y los estresores psicosociales a los que se asocia, y que pueden mediar en la génesis del comportamiento autodestructivo, en comparación con víctimas de suicidio que no son médicos. Gold, Sen y Schwenk (2013) han estudiado los datos del Registro de Muerte Violenta en 17 estados de los Estados Unidos entre 2003 y 2008, con una muestra de 31.636 adultos víctimas de suicidio de los que 203 son identificados como médicos. De los resultados destaca que no existían diferencias significativas en la prevalencia de trastornos mentales actuales claramente identificables entre médicos y no médicos (46\% versus $41 \%$ ) ni entre trastornos depresivos actuales (42\% versus 39\%). Los médicos tienen menos problemas de alcohol y dependencia de otras sustancias ( $14 \%$ versus $23 \%, \mathrm{P}=$. o04). Al evaluar el número total de trastornos mentales conocidos por cada persona suicidada, resulta que dos tercios de todas las víctimas tenían al menos un trastorno, 31\% tenían dos, y el 6\% tenían 3 trastornos mentales; sin diferencias significativas entre médicos y no-médicos. Para los médicos, las armas de fuego son también el método más común de suicidio (48\%), seguido de envenenamiento $(23,5 \%)$, precipitación $(14,5 \%)$ y por ahorcamiento (14\%).Los suicidas que no eran médicos usaron las armas de fuego en primer lugar (54\%), seguido por la ahorcamiento (22\%), seguido por el envenenamiento (18\%) y la precipitación (Gold, Sen, Schwenk 2013). En el análisis de regresión logística, estos autores obtienen un modelo en el que el tipo de trabajo (médico o no-médico) estaba en función de variables demográficas de las víctimas (edad avanzada, OR 1.04, CI:1.03-1.05, P<.0005), de variables de salud mental (tener una enfermedad mental conocida, OR1.34, CI.1.01-1.82, $\mathrm{P}=.045)$ y de estresores psicosociales identificables, como tener un problema laboral (OR 3.12, CI:2.10-4.63, P<.0005), en mucha mayor medida que otro estresores psicosociales no relacionados con el trabajo. La insatisfacción con el trabajo parece mediar de forma causal con depresión y suicidio entre los médicos. A casi tres cuartas partes de todas las víctimas de suicidio se les pudieron realizar análisis toxicológicos para identificar medicamentos y otras sustancias en los tejidos corporales, resultando positivos en el $27 \%$ de ellas. Los médicos tenían mayor probabilidad de dar resultado positivo que los controles para antipsicóticos (OR 28.7, CI: 7.94-103.9, P<.0005), benzodiacepinas (OR:39.5, CI:15.8-99.0, P<.0005). No se encontraron diferencias significativas para antidepresivos, opiáceos, anfetaminas o cocaína.

Podemos concluir que el suicidio de los médicos difiere del de la población general en varios aspectos. En los médicos están presentes de forma significativa los problemas laborales, frente a otros estresores como muerte de familiares o allegados y crisis personales recientes; lo que puede reflejar la naturaleza intrínsecamente estresante de la actividad clínica, así como la falta de recursos para hacerles frente de forma eficaz. Por otra parte, parece que la identidad del médico está demasiado centrada en su rol profesional, lo que puede hacerle más vulnerable a sufrir un mayor impacto por los problemas laborales. Entre ellos se conoce el papel mediador de variables ocupacionales tales como la falta de control sobre las condiciones de trabajo, los conflictos de rol, las demandas de los pacientes, experiencias degradantes o acoso, el burnout y los conflictos con los colaboradores, como factores significativos de riesgo para la ideación suicida en médicos (Lindfors et al. 2009, Fridner et al. 2011, Wada et al. 2011, Bovier et al. 2009).

Varios estudios han mostrado la importancia del efecto de la depresión no bien tratada en el riesgo de suicidio, especialmente en médicos mujeres, aunque diferentes estudios constatan la existencia de similares riesgos psiquiátricos para médicos y no-médicos. (Center et al. 2003, Gagne et al. 2011, Frank, Dingle 1999).

Los médicos tienen mayores resistencias para pedir ayuda profesional por estigma que han internalizado a lo largo de su formación y al temor de que les perjudique en su 
carrera, de forma que sólo la mitad de los médicos se muestran dispuestos a buscar ayuda profesional si llegaran a desarrollar una enfermedad mental (Hassan et al 2009).

\section{FACTORES ETIOPATOGÉNICOS QUE PUEDEN EXPLICAR EL INCREMENTO DE RIESGO DE SUICIDIO EN LOS MÉDICOS}

\section{Riesgos psicosociales inherentes al trabajo del médico, como el estrés propio de la actividad clínica, el acoso y el desgaste profesional en el trabajo}

El ambiente laboral se refiere a las características del medio laboral, tales como las características físicas, las políticas organizativas y los comportamientos característicos de las personas en el trabajo. Algunos medios de trabajo promueven la moral y la productividad del empleado; los trabajos proporcionan estructura y sentido a las vidas de los empleados, sensación de satisfacción y logro, y una base para la autoestima y la identidad personal. Otros ambientes de trabajo median en insatisfacción, y desesperanza, frustración, aislamiento y gran presión, como por la rigidez y la falta de una clara dirección y participación.

En la actualidad se están produciendo una serie de cambios radicales en los servicios sanitarios, en el sentido de que la eficiencia y el coste-efectividad son las herramientas más valoradas para determinar la financiación de los proyectos y la reestructuración de los servicios, e incluso la calidad asistencial y la seguridad de usuarios y sanitarios. Es evidente que estos cambios van a reducir la satisfacción laboral y van a condicionar más problemas de salud mental en los profesionales sanitarios, con mayor riesgo de burnout y depresión (Chan, Huak 2004).

En comparación con los médicos de los servicios centrales, los clínicos de hospital general presentan mayores niveles de estrés laboral, menor satisfacción, y menor calidad de vida/satisfacción general, que además disminuye con la edad, así como la autoeficacia percibida por los médicos, a la vez que aumenta la atribución de control externo en el trabajo, lo que se explica por el efecto del estrés profesional acumulado a lo largo del tiempo por desajuste persistente entre el esfuerzo realizado y el refuerzo percibido (Spector 1982; Gist, Mitchell 1992; Mingote 1995).

Una de las primeras encuestas realizadas a 850 médicos de familia por la Academia Americana de Medicina ilustra la vulnerabilidad al estrés de los médicos de atención primaria, de forma que el $65 \%$ de ellos reconocen un elevado nivel de estrés laboral, determinado por la sobrecarga de trabajo clínico, la presión de tiempo, los costes de los seguros y las interferencias de las regulaciones gerenciales, el trabajo administrativo y la necesidad de tratar problemas emocionales para los que no están formados (American Academy of Family Physicians 1979).

Los estudios de Revicki y May (1983, 1985), May y Revicki (1985) y Revicki et al. (1991) con una muestra de 320 médicos Carolina del Norte constatan específicamente que el estrés ocupacional crónico del médico ejerce un efecto directo sobre el desarrollo de síntomas depresivos y aumenta el riesgo de depresión, moderado directamente por el apoyo sociofamiliar y emocional, e indirectamente por la influencia del locus de control sobre la percepción del estrés. Los médicos más estresados tienen menor percepción de control, menor apoyo social percibido y locus de control externo, mientras que la atribución de control interno es un factor positivo en el afrontamiento efectivo del estrés profesional. El 5\% de los médicos presentaba síntomas depresivos en un rango clínico y el 30\% en rango leve-moderado (según la escala autoaplicada de Zung), tasas similares a las de la población general (Boyd \& Weissman 1981). Los médicos con mayor percepción de control personal y apoyo de familiares, colegas y supervisores presentan menores niveles de estrés ocupacional y de síntomas depresivos. Los lazos familiares positivos y el apoyo percibido de los compañeros moderan el estrés percibido por los médicos, aunque cuando este es elevado puede interferir en la vida familiar tanto como en la propia salud. 
Estos mismos autores han desarrollado un Cuestionario de Estrés en el Médico de 26 ítems y 4 factores: estrés profesional intrínseco, productividad percibida, interferencia con la vida familiar y estrés profesional externo que tiene unas buenas propiedades psicométricas de fiabilidad y validez (Revicki y May 1991) que he utilizado en mi estudio doctoral (Mingote 1995). Revicki et al. (1993) estudiaron el estrés laboral, el apoyo social y los niveles de síntomas depresivos en médicos residentes a lo largo de 3 años: Concluyeron que la ambigüedad de rol se asoció con la intensidad de los síntomas depresivos a lo largo del tiempo.

Según el modelo de Karasek y cols. (1979), el trabajo del médico está incluido en el nivel superior de calidad ocupacional, un "trabajo activo", que proporciona más satisfacciones que estrés laboral, pues incluye elevadas demandas psicológicas (de conocimientos, de rendimientos y con relaciones interpersonales) con oportunidades para ejercer un control profesional eficaz, a través de la adquisición de nuevos conocimientos y habilidades personales. Kauppinen-Toropainen y cols. (1983) han confirmado este modelo en una muestra de 7000 trabajadores representativos de Finlandia, de forma que lo fundamental para disminuir el estrés y mejorar la satisfacción y la productividad es poder disponer de los adecuados recursos de control individual y colectivo sobre las demandas psicológicas del trabajo. Para ello es necesario asegurar la competencia y la autonomía profesional eficaz en cuanto a la toma de decisiones clínicas. La pérdida de control sobre el proceso de toma de decisiones clínicas por fuerzas externas como de tipo económico-gerencial, asociadas al proceso de burocratización de la práctica clínica moderna, las denuncias por mala praxis y los dilemas éticos son importantes fuentes de estrés para el médico. Deberíamos prestar más atención al mantenimiento de los valores profesionales y deontológicos, así como a la construcción de un clima afectivo y efectivo de trabajo entre todos los profesionales sanitarios.

Con el modelo de Siegrist y Theorell, Siegrist y Rödel (2006) el estrés en el trabajo se concibe como un desequilibrio persistente entre esfuerzo y refuerzo percibido revisando 46 estudios: En general los resultados apoyan la hipótesis de una asociación consistente entre estrés laboral y conductas de riesgo para la salud, tales como el abuso de alcohol y otras sustancias de consumo.

Como el burnout, el acoso laboral, relacionado con unas condiciones laborales insanas median en el riesgo de tener ideas de suicidio en médicos varones de hospital en Suecia y en Italia, según los resultados del estudio sobre la salud y la organización en los Médicos de Hospital en Europa (HOUPE) con una muestra de 697 médicos: el 12\% de los médicos de ambos países reconocieron tener ideas de suicidio recientes, que relacionaban con el hecho de haber sido expuestos a experiencias degradantes, acoso laboral, conflicto de rol, tener escaso control sobre el trabajo y escaso apoyo laboral, como este mismo grupo ya lo había demostrado en mujeres médico. El ámbito académico médico es muy competitivo, con intensa rivalidad entre iguales por la financiación, publicar, invitaciones y otros premios (Fridner et al 2011).

\section{Mal afrontamiento de los riesgos psicosociales inherentes a la actividad clínica, por insuficiencia de recursos para el afrontamiento de los mismos}

Según un estudio publicado por Blumenthal et al. (2006) el 10\% de los médicos residentes en Estados Unidos al finalizar su formación se declaran incapaces de manejar el tratamiento de procesos como la depresión, el abuso de drogas, el consejo al paciente y la violencia domestica, a pesar de que su competencia clínica general es considerada satisfactoria. El trabajo contiene datos de 4832 residentes pertenecientes a diferentes especialidades médicas. Estos resultados alertan sobre las limitaciones de los actuales programas de formación de grado y de postgrado, e indican la necesidad de implementar la formación en conocimientos y habilidades psicosociales de los médicos para hacer frente a los retos de la práctica clínica. Heim (1991) estudia los factores estresantes y los estilos de afrontamiento de 1600 médicos suizos, y resalta que son las mismas variables ocupacionales las que median en satisfacción y/o en estrés 
laboral, según como se perciban las relaciones con los enfermos y con los miembros del equipo asistencial, las condiciones trabajo, el salario, etc. Inicialmente los médicos intentan minimizar las consecuencias de los estresores de la actividad clínica, hasta que se va produciendo una gradual internalización y toma de conciencia de las dificultades y conflictos situacionales, lo que media en la búsqueda y selección de soluciones a los mismos, sean adaptativas o no. Realizar un esfuerzo reflexivo, el estudio, la búsqueda de apoyo social y el sentido del humor son estrategias proactivas de afrontamiento adaptativo. En un $20 \%$ de la muestra predominan mecanismos desadaptativos como el aislamiento social, la evitación-negación persistente de los problemas y el autotratamiento patológico con alcohol y otras sustancias de abuso. Según el modelo de Lazarus y Folkman (1984) y Moos \& Billings (1982) para el afrontamiento del estrés laboral, podemos emplear tres categorías de afrontamiento: control, que incluye tanto acciones como reevaluaciones cognitivo-afectivas que son proactivas y promueven el aprendizaje (adaptativas, funcionales), evitación-escape de la realidad, y alivio de los síntomas consecuencia o producto del estrés laboral, que pueden ser saludables (como el entrenamiento en relajación) o patológicas y patógenas, como el abuso de alcohol y otras sustancias de consumo. Las estrategias incluidas dentro de cada grupo son muy heterogéneas, y además pueden mediar en resultados adaptativos o desadaptativos diferentes, según otras variables temporales, personales y contextuales diferentes. En general, la identificación, comprensión y expresión adecuada de las emociones experimentadas asociadas a situaciones estresantes es adaptativa, mientras que la inhibición-bloqueo emocional es patológica y media en el padecimiento de enfermedad mental. El apoyo social eficaz modera la percepción de estrés y el riesgo de padecer alteraciones psicopatológicas y depresión de forma parcial. Desde la teoría cognitiva de la atribución, el control percibido y la capacidad para ejercer control, conceptualizada por Rotter (1966) y desarrollada en el ámbito sociolaboral por autores como Bandura (1977) y Spector (1988), la atribución de control se adquiere por aprendizaje a partir de la experiencia de refuerzos positivos y negativos. El "lugar de control" se refiere a la atribución, expectativa e interpretación que hace una persona sobre las contingencias que determinan su conducta, en función de las experiencias previas. Se trata de una variable dinámica, las puntuaciones de "internalidad" aumentan normalmente con la edad, a través de la adquisición de nuevos aprendizajes y de una mayor autonomía económica, laboral y personal. La exposición del sujeto a situaciones de incontrolabilidad aumenta su "externalidad", disminuye su autoestima y autoeficacia o maestría personal, factor de riesgo para experimentar síntomas de ansiedad y depresión (Spector 1988). Según este autor, el "lugar de control" en el trabajo es una importante variable mediadora de la conducta en las organizaciones, relacionada con la motivación, las actitudes, el esfuerzo, el rendimiento y la satisfacción laboral. Internalidad se asocia a mayor satisfacción, implicación-compromiso laboral (componente benigno del Patrón de Conducta Tipo A), mayor satisfacción y menor estrés laboral, y mejor calidad de vida, a través de realizar un renovado esfuerzo para adquirir mayor información y controlar las demandas laborales. Al contrario "externalidad" se asocia a la experiencia de frustración de expectativas y objetivos previos, por la intervención de fuerzas ajenas a ellos mismos, como decisiones gerenciales-económicas, ausencia de refuerzos contingentes con el esfuerzo, y en general por dificultades crónicas acumuladas a lo largo del tiempo. En general, es deseable que las personas tengan cierta percepción de control sobre sus vidas, como consecuencia de un protagonismo personal responsable, sea por control conductual, cognitivo, decisional, informacional y/o retrospectivo, con un efecto beneficioso para la reducción del estrés.

En el estudio de Mingote (1995) con una muestra representativa de médicos de hospital general, destaca que los médicos clínicos tengan mayor nivel de estrés laboral y menor satisfacción con su trabajo que los que realizan su actividad sin tener contacto directo con los pacientes, y reconocen peor apoyo social en el trabajo, en relación con las mayores complejidades y demandas psicológicas de la actividad clínica que la técnica, sobre todo por no disponer de los recursos adecuados para el afrontamiento del estrés interpersonal inherente al rol clínico profesional. El estrés laboral del médico clínico puede llegar a comprometer la eficacia de las organizaciones sanitarias, la seguridad de 
los usuarios y de los profesionales sanitarios. Además, a mayor edad el médico está más insatisfecho con su trabajo, se percibe con menos autoeficacia o maestría percibida, y con una clara "externalidad" frente a los médicos más jóvenes. La atribución de control externo se asocia de forma positiva con mayor nivel de estrés laboral y despersonalización de las relaciones asistenciales. La externalidad indica que globalmente los médicos de la muestra creen que tienen poca capacidad de control sobre las condiciones y los resultados de su trabajo. La disminución significativa de la satisfacción laboral y de la autoeficacia percibida, así como el aumento de la externalidad de los médicos de más edad, son importantes indicadores psicométricos de la falta de la autonomía profesional y del riesgo para la salud de los médicos y para el propio sistema sanitario, por lo que se deben implementar medidas eficaces para la participación de los médicos en la planificación, regulación y control de la organización y la gestión de los centros sanitarios. Por ello es fundamental instaurar procedimientos válidos y fiables para mejorar la participación de la Organización Médica Colegial en la regulación de la práctica clínica, según la evidencia científica y el consenso deliberativo y democrático, garantizar el aprendizaje continuado compartido, y el desarrollo efectivo de la carrera profesional. El estrés laboral de los médicos depende de variables ocupacionales como especialidad y número de horas de trabajo a la semana, así como de variables individuales, como Patrón de Conducta Tipo A Impaciencia y hostilidad, así como de mayor despersonalización de las relaciones asistenciales y peor apoyo social percibido. La calidad de vida de los médicos se asocia positivamente con satisfacción laboral, optimismo y maestría, y negativamente con estrés laboral, despersonalización y Patrón de Conducta Tipo A-impaciencia (Mingote 1995).

Dada la importancia del estrés interpersonal en el rol profesional del médico, tiene un especial interés la aplicación de la teoría interpersonal del suicidio (Joiner 2009) para la prevención del suicidio en médicos a través de la intervención de Cornette el al (2009) en Wisconsin. Según esta teoría, tres factores aumentan el riesgo de suicidio: sentirse una carga para otros (ineficacia, inefectividad, incapacidad de control), fracasar en la consecución del sentimiento de pertenencia a un grupo de iguales respetables (aislamiento social, desesperanza) y capacidad adquirida para consumarlo a través de la experiencia de un dolor emocional generalizado, en grupos de riesgo de suicidio por padecer varios trastornos mentales: trastornos depresivos, trastornos por uso de sustancias y alcohol, trastorno de personalidad borderline, trastornos de ansiedad, anorexia nerviosa y esquizofrenia.

En cuanto a la psicología del comportamiento suicida, se comprobó que los pacientes suicidas compartían las características cognitivas de los deprimidos con una alta incidencia de pensamientos negativos complicada por errores de pensamiento lógico (generalización excesiva, catastrofismo, pensamiento en blanco y negro) y la tendencia a que los acontecimientos vitales desencadenaran a largo plazo un determinado esquema de creencias. Beck demostró que las conductas suicidas se asocian también con desesperanza en cuanto al futuro. En la actualidad, el modelo más relevante es el que hace referencia a la sensación de "sentirse atrapado", de que no hay escape, no es posible la huida y ello supone un patrón determinado en cuanto al modo de procesar la información sobre uno mismo y sobre el mundo. Puede definirse como la incapacidad, o la incapacidad percibida, de escapar de un entorno adverso después de haber sufrido una derrota, una pérdida o humillación. Los enfoques basados en resolución de problemas pueden no ser muy útiles si no se tiene claro como surgen y se perpetúan los déficits y si la rumiación y la desesperanza van en aumento.

Tres características cognitivas diferencian a los individuos deprimidos suicidas de los que no lo son: 1) Hipersensibilidad a acontecimientos vitales relacionados con derrotas, con sentirse "perdedor" o humillado. 2) "No tener escapatoria" en el sentido de sentirse atrapado. Se debe a déficits en la capacidad de resolución de problemas y se pone de manifiesto en la confrontación de los estímulos citados anteriormente. 3) No tener posibilidad de ser rescatado, pensar que la situación continuará indefinidamente, mediado por procesos cognitivos deficientes que llevan a la desesperanza con respecto al futuro. 
Todo ello supone que se debe prestar especial atención a eventos que puedan resultar particularmente humillantes o hacer que una persona se sienta vencida o atrapada, ya que pueden ser particularmente importantes en la cadena causal y tener en cuenta el efecto de un estado de ánimo deprimido en un sistema cognitivo vulnerable (Williams, Pollock 2000).

\section{Especiales dificultades de los médicos para asumir el rol de enfermo}

Ser "pacientes especiales", eufemismo de "los peores enfermos", se asocia a recibir peor tratamiento y peor seguimiento que el resto de los pacientes, y que habitualmente buscan ayuda profesional demasiado tarde, cuando el trastorno mental se ha cronificado, agravado y complicado con otros trastornos médicos y enfermedades físicas, por lo que tienen peor pronóstico general. Cuidar a los demás de forma prolongada no debe asociarse con insensibilidad y negligencia respecto de la propia salud. Además, la bata blanca no nos protege frente a la depresión: La depresión es al menos tan prevalente entre los médicos como en la población general. La prevalencia-vida de síntomas de depresión clínica autoevaluada es del $12,8 \%$ en un estudio prospectivo de más de 1300 médicos seguidos entre 1948 y 1964 (Ford et al 1998), es casi idéntica al 12\% de depresión mayor en varones en EE.UU. La única diferencia es la edad posterior de comienzo en los médicos (Shafer, Craft 1999). La prevalencia-vida de síntomas autoidentificados de depresión en médicas de 19,5\% en el Estudio de la Salud de Mujeres Médicos ( $=4501)$, es comparable a la de la población general y mujeres profesionales (Frank, Dingle 1999). Pero los médicos se resisten a buscar ayuda profesional por temor al estigma social y comprometer su carrera profesional, por lo que la posponen hasta que ya el trastorno mental se ha cronificado y complicado con otras patologías graves y ha afectado negativamente a sus pacientes. Más allá del impacto en su salud personal, es preciso tener en cuenta el riesgo de afectar a la calidad asistencial y arriesgar la seguridad de los pacientes que atienden. Diferentes estudios concluyen que los médicos fracasan en detectar o tratar entre el $40 \%$ y el $60 \%$ de los casos de depresión. Igualmente, en el médico enfermo es muy frecuente el autodiagnóstico, la autoprescripción, el conseguir consultas "informales" e infrecuentes, y la asunción del estado de "VIP". El estigma inherente al padecimiento de una enfermedad mental es internalizado también por los médicos a lo largo de su carrera profesional a través de un aprendizaje activo, con intenso temor al desprestigio y al daño profesional y social, con numerosos estereotipos negativos y erróneos que conforman la construcción interna de actitudes y comportamientos antidiscriminación que se concretan en la autoprescripción y la evitación del rol de enfermo, como si fuese incompatible con el de médico, por ser tan reacios a admitir el padecimiento de una enfermedad mental. El estigma supone la pérdida de estatus social y la discriminación desencadenada por estereotipos negativos sobre las personas etiquetadas como "enfermos mentales (Ritsher, Otilingam, Grajales 2003). El incremento gradual de síntomas depresivos y de ansiedad puede mediar en sucesivos intentos de auto-tratamiento patológico (automedicación, alcoholismo, adicciones), auto-negligencia, déficits cognitivos, deterioro de la salud propia y riesgo para terceros en el ejercicio de su profesión. A menudo las personas que sufren un trastorno mental posponen más de un decenio la solicitud de tratamiento, cuando ya es más grave y se ha complicado con otros padecimientos físicos y mentales. Los síntomas depresivos ocurren a lo largo de un continuum más que en categorías discretas, con un importante impacto en el funcionamiento a lo largo del tiempo; mientras que el tratamiento precoz eficaz puede prevenir la progresión de la enfermedad a estadios clínicos subsiguientes. Estos datos son consistentes con un proceso de sensibilización al estrés, de forma que los sucesivos episodios pueden desencadenarse tras mínimos estresores, o incluso sin ninguno de ellos (Post, 2012).

El rol de médico no proporciona protección contra la enfermedad. Los factores que predisponen a los médicos al acto suicida están relacionados con el acceso y dependencia a las drogas, las tensiones derivadas de la profesión y los conflictos a nivel personal y social. Igualmente de la Cruz, Corominas y Sarró (1988) explicitan:"La detección y tratamiento de los trastornos mentales de los profesionales de la medicina es difícil. Los 
médicos pueden ser bastante tolerantes con las alteraciones emocionales de sus compañeros, con una fuerte resistencia a intervenir en los problemas considerados personales". Igualmente, la Asociación Médica Americana (1973) denunció la apatía, el desconocimiento y la falta de responsabilidad general de los médicos respecto del médico deteriorado e incompetente, por lo que la intervención que se toma suele llegar demasiado tarde en el curso de los hechos, incluso tras el suicidio del médico.

\section{Disociación patológica entre el self público y el self privado. El mantenimiento del "self público" correspondiente a la "bata blanca", puede asociarse a un daño grave en el "self privado"}

La estigmatización de las personas con enfermedad mental constituye un importante problema de salud pública que demanda la realización de programas anti-estigma dirigidos a médicos y a otros sanitarios para mejorar la atención al enfermo mental. Los médicos deben ser advertidos contra la tendencia defensiva a refugiarse en el rol profesional para autoinducirse un "sentimiento de superioridad" sobrecompensador de la "debilidad" de tener los mismos problemas que las personas normales. Esta compensación defensiva puede llegar a la arrogancia narcisista y al "complejo de Dios" del médico (descrito por Jones en 1913), con sentimientos irrealistas de superioridad como expresión de un persistente autoengaño patológico, que le hace vulnerable a ulteriores riesgos psicosociales y crisis más graves. El médico satisfecho con su actividad profesional, puede mantener una adecuada disponibilidad emocional con los pacientes, como con su propia familia, en contacto con sus propias emociones, en vez de ignorar el propio malestar. Se trata de conseguir que los trastornos mentales sean reconocidos como enfermedades muy prevalentes y con características similares a otras que se abordan de forma eficaz en Medicina. Entre los médicos es especialmente importante asegurar una ayuda profesional eficaz y confidencial, que garantice su seguridad, la de los pacientes que atienden y de sus compañeros de trabajo. Los Colegios de Médicos deben funcionar como grupos eficaces de apoyo profesional.

\section{Especiales dificultades del tratamiento de los médicos por otros médicos}

Tratar médicos con trastornos mentales puede constituir una experiencia muy satisfactoria, pero que implica también una especial responsabilidad, así como mayores reacciones contratransferenciales, con sentimientos intensos que pueden comprometer la neutralidad terapéutica y distorsionar el juicio clínico, minimizar la gravedad de los problemas que padecen e indicar tratamientos subóptimos que contribuyen a que tengan evoluciones peores que los pacientes que no son médicos. El tratamiento de los trastornos mentales en médicos, constituye un verdadero reto profesional para los psiquiatras, quienes experimentan varios sesgos cognitivo-afectivos con los colegas, (como por escotomización, minimización o bien magnificación, etc.) por los que les tratan de forma menos adecuada que al enfermo común. El estrés interpersonal del médico se produce por identificación (complementaria o concordante) con la angustia y las defensas del enfermo.

Probablemente, la renuencia de los médicos a intervenir en el caso de un colega deteriorado, sea una predecible consecuencia de su propia negligencia respecto a sus necesidades y conflictos emocionales, así como por una mayor identificación con su malestar emocional. Por estas dificultades y el riesgo de mala praxis se han creado programas específicos para la prevención y tratamiento de los médicos enfermos, primero en Estados Unidos por la Asociación Médica Americana (desde 1975) y que luego se han generalizado en los distintos países.

\section{Litigios por mala praxis}

Se asocia fuertemente con burnout $(\mathrm{p}<0.0001)$, depresión $(\mathrm{p}<0.0001)$ y con ideas de suicidio $(\mathrm{p}<0.0001)$ entre cirujanos en Estados Unidos. En análisis multivariantes, tanto depresión como burnout se asocian independientemente con una reciente denuncia de 
mala praxis. El número de horas trabajadas, de guardias, la subespecialidad, y el medio laboral se asocian también con una denuncia reciente (Shanafelt, Balch, Dyrbye et al. 2011). La ideación suicida aumentó notablemente en los médicos durante los 3 meses siguientes de haber cometido un error grave en su práctica profesional. En el estudio de Lindeman et al (1997) los médicos finlandeses que estaban bajo vigilancia médica oficial presentaban un riesgo significativamente mayor de suicidio consumado.

\section{¿Por qué las médicas tienen más riesgo de suicidio que sus compañeros y que las mujeres de la población general?}

Algunos factores pueden ser específicos de la actividad clínica, mientras que otros corresponden a variables biológicas y psicosociales. Así Rose y Rosow (1973) hallaron que otras trabajadoras sanitarias en contacto directo con pacientes tienen un riesgo doble de cometer suicidio que los controles, sugiriendo que esta faceta de la actividad profesional de las doctoras puede ser una parte importante de la etiología del mismo. Varios factores de vulnerabilidad previa pueden explicar parte de estas elevadas tasas, como la existencia de morbilidad psiquiátrica concurrente, sobre todo depresión en el $75 \%$ de las doctoras que se suicidan (Ross 1973, Barraclough et al., 1974).

1. En el primer aspecto, las médicas tienen 1,6 veces más riesgo de presentar burnout en comparación con sus colegas varones, lo que se asocia a falta de autonomía y control percibido sobre el trabajo, menos recursos de apoyo y menor salario que los médicos. Las médicas están más satisfechas con su especialidad, la relación con el paciente y con sus compañeros en comparación con estos, dedican más tiempo a la asistencia, ven más enfermas y más pacientes con problemas psicosociales complejos (Mc Murray et al. 2000). Otro estudio realizado con 420 médicos estadounidenses de atención primaria, concluyó también que las médicas tienen un riesgo doble de reconocer elevados niveles de estrés y burnout en comparación con sus compañeros (Croasdale 2005). Resultados análogos se obtuvieron de un estudio de 2.398 médicos canadienses sobre sus prácticas y actitudes hacia la asistencia. Las médicas prefieren trabajar en organizaciones públicas y equipos de trabajo, mejor que la práctica individual; mientras que los médicos están más inclinados a trabajar en un tipo de asistencia individual. Como en otros estudios, se constata que los ingresos de las médicas es inferior al de sus colegas varones, además de sufrir mayores niveles de acoso laboral, sobre todo durante el periodo de formación, que sus compañeros varones.

2. En el estudio presentado en el Congreso de la Asociación Americana de Medicina (2005), Horner-Ibler comunicó los resultados de una encuesta anónima realizada a 420 médicos de atención primaria y a 2.500 de sus pacientes, según la cual las médicas presentan una tasa doble de niveles elevados de estrés y de burnout, en comparación con sus compañeros varones. Ellas también expresaban su deseo de tener más tiempo para atender a los pacientes con patologías complejas, deseaban lugares de trabajo más saludables, y se sentían más enfrentadas con los valores de las organizaciones en las que trabajaban, respecto sus compañeros varones (tomado de Croasdale 2005).

3. Tanto en Suecia como en Italia, varios estresores del trabajo aumentan el riesgo de suicidio en médicos mujeres. Según el estudio HOUPE de Fridner et al. (2009) el 13,7\% y el 14,3\% de las médicas de Suecia e Italia reconocieron ideas de suicidio en los últimos 12 meses. Entre las médicas de Suecia las idas de suicidio estaban determinadas por dos variables independientes relacionadas con el trabajo: experiencias degradantes y acoso en el trabajo (OR 3,03, 95\% CI, 1,48-6,23), así como la autoprescripción. En las médicas de Italia destacaban las variables asignar un trabajo sin los recursos adecuados, así como ser joven y padecer burnout o depresión. Se concluye destacando la importancia del control de los riesgos psicosociales en el ámbito sanitario.

4. En parte también, las mujeres presentan mayor prevalencia de depresión que los hombres. Los trastornos afectivos constituyen el grupo diagnóstico con mayor 
riesgo de conductas suicidas. El riesgo de consumación del suicidio entre los pacientes depresivos a lo largo de la vida es de un 15\%, 25 veces mayor que en población general. Existe una elevada tasa de suicidio entre los pacientes con trastornos afectivos graves, como con sintomatología delirante, mayor desesperanza y hostilidad, de forma que hasta uno de cada 6 de ellos se provocan la muerte. Los médicos de ambos sexos que se suicidan padecen un trastorno del estado de ánimo y/o dependencia de alcohol o de otras sustancias de consumo, así como conflictos y dificultades profesionales o familiares. El mal afrontamiento de estos problemas de salud y el acceso a drogas constituyen importantes factores mediadores de las conductas autodestructivas (Mingote, Jiménez Arriero, Osorio Suárez, Palomo 2004).

\section{¿Por qué los médicos se suicidan, en mayor medida que otros profesionales?}

“Teniendo tanto, ¿por qué podría un médico quitarse la vida? Central a la incredulidad es que uno de los principales dogmas de la vocación médica es preservar y proteger la vida. ¿No es por esto por lo que hombres y mujeres se hacen médicos?" (Myers y Gabbard 2008).

El suicidio se entiende actualmente como un fenómeno complejo que resulta de la interacción de una serie de factores biológicos, genéticos, psicológicos sociológicos y ambientales.

Los factores que pueden contribuir al suicidio pueden clasificarse como estadodependientes o carácter-dependientes, o como distales (carga genética, características de personalidad, escaso crecimiento fetal, problemas perinatales, experiencias tempranas traumáticas, trastornos neurobiológicos como disfunción del sistema serotoninérgico y noradrenérgico, hiperactividad del eje hipotálamo-pituitaria-adrenal), o proximales (trastornos mentales, problemas físicos, crisis psicosociales, acceso a medios, exposición, problemas jurídicos o relacionados co el trabajo). La relación entre los factores de riesgo puede describirse mediante modelos como el de Estrés-Diátesis de Hawton, adaptado de Mann, (2003) que es compatible con modelos actuales sobre la interacción dinámica continua entre genes y entorno a lo largo del ciclo vital. Las crisis agudas y los problemas psiquiátricos son con frecuencia los estresores proximales que desencadenan el comportamiento suicida mientras que el pesimismo y la desesperanza o la agresividad y la impulsividad son componentes de la diátesis. Los factores genéticos, las experiencias de la infancia y otros factores como los niveles de colesterol también afectan a la diátesis. El modelo estrés-diátesis hace referencia a la relación entre factores de riesgo de suicidio que pueden clasificarse como estado-dependientes o de carácter genético; o como proximales o distales. Las crisis emocionales o psicosociales y los trastornos psiquiátricos suelen ser los factores proximales que generan estrés, mientras que el pesimismo, la desesperanza, la agresividad y la impulsividad son componentes de la diátesis que da lugar a conductas suicidas. Factores genéticos o familiares, experiencias infantiles, y otros factores, incluidos los niveles de colesterol, también afectan a la diátesis. El modelo estrés-diátesis es compatible con otros modelos recientes sobre la interacción entre genes y entorno, pero se necesitan ulteriores estudios para evaluar su capacidad de predicción.

Según los estudios epidemiológicos disponibles, cerca del 90\% de las personas que se suicidan padecen algún tipo de trastorno mental, aunque no estuviese diagnosticado previamente. Se trata de médicos que nunca han sido valorados por un psiquiatra, ni han recibido tratamiento profesional, aunque a menudo han intentado tratarse a si mismos.

Entre los principales factores de riesgo se incluyen la disponibilidad de medios de suicidio (como el acceso a drogas) y los conocimientos y habilidades técnicas que aseguran la eficacia del método de suicidio empleado pueden ser relevantes. También pueden influir algunas características de personalidad de los médicos, como las de tipo obsesivo-compulsivo, propensas a la depresión, junto con altas tasas de abuso de alcohol y drogas, y el estrés ocupacional propio de los médicos. 
Silverman (2000) revisó la investigación disponible y concluyó que existen varios factores adicionales específicos que incrementan el riesgo de suicidio en los médicos, como son:

1. Factores socio-demográficos: edad superior a 45 años en las médicas y a 50 años en los médicos; y permanecer soltero, separado, divorciado, o con ruptura marital actual.

2. Mal estado de salud, sea por hábitos tóxicos (abuso de alcohol y otras drogas), trastornos mentales (especialmente ansiedad, depresión y trastornos de personalidad) y/o enfermedades médicas graves, debilitantes o que producen dolor crónico. La patología dual es muy común en médicos, en especial la peligrosa combinación de trastornos del estado de ánimo y adictivos.

3. Factores profesionales: Amenaza al status, autonomía, seguridad, estabilidad económica, pérdidas recientes o mayores demandas laborales.

4. Acceso a medios autolíticos, como medicaciones letales.

Myers y Gabbard (2008) destacan los siguientes aspectos a tener en cuenta para valorar el riesgo de suicidio en médicos:

- Antecedentes personales de episodio depresivo y de intento de suicidio. Los intentos previos constituyen el factor de riesgo más significativo.

- Antecedentes familiares de trastornos depresivos y de suicidio.

- Aislamiento profesional, falta de apoyo socio-familiar y de otros factores de protección, como el trabajo en equipo y la supervisión.

- Ser investigado por mala praxis o incompetencia profesional, puede constituir una importante injuria narcisista, sobre todo en personas perfeccionistas y con una mala regulación de la autoestima.

- Mala adherencia terapéutica.

- Trastornos mentales graves, crónicos y refractarios al tratamiento, que inducen desesperanza y un importante estigma internalizado, además del estigma cultural, que refuerzan la negación de la enfermedad, la autoprescripción de sustancias, e incrementan el riesgo de suicidio en médicos.

- Afecciones orgánicas dolorosas, debilitantes o de mal pronóstico.

Según Myers y Gabbard (2008) no existe una única forma de presentación del médico-enfermo con riesgo agudo de suicidio, pero destacan los siguientes síntomas cardinales: Elevados niveles de ansiedad, insomnio, falta de energía y fatiga, anorexia y pérdida de peso, enlentecimiento cognitivo, rumiaciones obsesivas, síntomas somáticos, desesperanza e ideas de suicidio (pasivos y activos).

\section{VALORACIÓN Y TRATAMIENTO DEL MÉDICO ENFERMO CON RIESGO DE SUICIDIO}

A pesar de que nuestra capacidad de predicción no es infalible, hay un número de datos clínicos que se asocian con riesgo de suicidio en nuestros pacientes" (OMS, World Health Report 2001).

Una de las principales normas de buena práctica clínica es no comprometer el juicio clínico porque el paciente sea médico. Existe una larga historia de médicos suicidados que no fueron diagnosticados ni tratados adecuadamente a causa de sus propias resistencias y la connivencia de colegas complacientes.

Myers y Gabbard (2008) nos proponen los siguientes principios básicos a tener en cuenta cuando se tratan médicos:

1. Trata de valorar a los médicos lo más rápidamente posible, en especial cuando son ellos mismos los que piden ayuda, ya que necesitan esta forma de cortesía profesional. 
2. Pregunta si dispone de medios y métodos de suicidio como clorato potásico, insulina, digoxina y fentanilo.

3. A menudo los médicos consultan "demasiado tarde y mal", cuando están muy enfermos, aunque lo nieguen, hasta poder llegar a sentirse irreparablemente dañado y una pesada carga para su familia. El o ella pueden llegar a ver su muerte como un alivio para su propia familia.

4. Asume que todos los médicos han contemplado el suicidio a lo largo de un continuum de intensidad y frecuencia, como forma de control último sobre la propia vida.

5. Cuenta con el hecho de que el médico-enfermo no sea sincero con su psiquiatra, para evitar la baja laboral y un ingreso psiquiátrico involuntario.

6. En este caso es esencial garantizar la privacidad y la confidencialidad del médicoenfermo.

7. Cuando sea posible, recuperar la profesión es a menudo un importante factor motivacional para hacer el tratamiento.

8. Trata las alteraciones del sueño con la medicación apropiada, por la estrecha asociación que existe entre estas y la conducta suicida.

9. Es esencial contar con la colaboración de familiares y/o otros profesionales, como los que le han tratado previamente, para poder realizar una evaluación completa, de forma comprensiva, persistente y exigente.

10. Un contrato terapéutico oral escrito, debe continuar a una cuidadosa valoración clínica.

Gabbard y Allison (2006) proponen los siguientes pasos en el tratamiento psicodinámico del paciente suicida y que igualmente se deben tener en cuenta en el caso de los médicos:

- Establecer una buena alianza terapéutica con el paciente en riesgo. El terapeuta debe explicitar que ni él ni nadie pueden impedir a un médico que se suicide, estableciendo una clara diferenciación entre las responsabilidades del paciente y del terapeuta dentro de los límites de la relación terapéutica.

- Establece el nivel de riesgo presente basalmente.

- Diferenciar entre fantasía de suicidio y la verdadera intención de llevar a efecto una verdadera conducta suicida.

- Investigar los acontecimientos precipitantes.

- Explora las fantasías del impacto interpersonal del suicidio.

- Monitoriza la transferencia y la contratransferencia. Los terapeutas que tratan pacientes suicidas, y especialmente si son médicos, suelen experimentar ansiedad, enfado, vergüenza y culpa cuando el paciente no mejora o quiere morirse, intenta suicidarse o muere por suicidio. En estos casos es muy útil y recomendable supervisar el caso y/o ponerse en tratamiento.

Maltsberger (2006) destaca algunos pasos fundamentales en el tratamiento ambulatorio de pacientes suicidas, especialmente relevantes para el tratamiento de médicos:

1. Completa una minuciosa evaluación del riesgo de suicidio desde el inicio y actualízalo cada día, con actitud comprometida, no neutral. Para ello es preciso ser capaz de contener la angustia de muerte que invade al paciente y trasmite al terapeuta.

2. Se debe conseguir un consentimiento informado y realizar un análisis riesgobeneficio en colaboración con el paciente.

3. Acepta sólo pacientes que quieren comprometerse en seguir vivos y dan una oportunidad al tratamiento. Maltsberger destaca la importancia de la relación real del terapeuta con el paciente, no la transferencia, como un "fuerte", un objeto seguro y confiable con el que identificarse. 
4. Asegura el tratamiento ingresado con poco tiempo de aviso.

5. Anota los recursos de apoyo con los que cuenta el paciente.

6. Asegura que existe un control externo del tratamiento del paciente.

7. Registra adecuadamente los datos precisos en la historia clínica.

\section{LA PREVENCION DEL SUICIDIO EN LOS MÉDICOS}

La prevención del suicidio en médicos presenta algunas peculiaridades pero también muchos aspectos comunes con los que se encuentran en la población general.

Cada cuarenta segundos fallece alguien por suicidio. Es la primera causa de muerte violenta en el mundo, se producen más muertes por suicidio que por la suma de homicidios y guerras, y es la décima causa de muerte globalmente. Las estimaciones realizadas indican que las víctimas podrían ascender de un millón a 1.5 millones al año en 2020 y que para entonces la depresión ocupará el segundo lugar en cuanto a carga por enfermedad en el mundo por lo que es necesario adoptar medidas coordinadas y más enérgicas para evitar ese número innecesario de víctimas, según la Organización Mundial de la Salud (OMS). Siguiendo sus recomendaciones, la prevención del suicidio está recibiendo cada vez mayor atención social ya que supone un serio problema de salud pública y muchos países están desarrollando estrategias nacionales de prevención. Se ha demostrado un aumento en el número de suicidios en los países desarrollados, en especial entre adolescentes y adultos jóvenes. Las recomendaciones de la OMS sobre Prevención del Suicidio están recogidas en una lista de publicaciones denominada genéricamente SUPRE (Suicide Prevention) entre las que se encuentran el Protocolo SUPRE-MISS (Multisite Intervention Study of Suicidal Behaviours) y una Guía para Médicos de Atención Primaria. Más recientemente también se ha publicado la Guía mhGAP concebida para proveer asistencia incluso en países que carecen de servicios de salud mental especializados.

Se acepta que los profesionales sanitarios juegan un importante papel en la prevención del suicidio, por lo que es importante identificar los grupos de riesgo, determinar las causas antecedentes y considerar las medidas prácticas de prevención. No obstante, algunos médicos no se sienten competentes para evaluar, diagnosticar y tratar a pacientes con depresión y riesgo suicida, por lo que es necesario que adquieran los conocimientos y las habilidades necesarias para la prevención del suicidio en las facultades de Medicina, en los periodos de especialización y a lo largo de su carrera profesional. El tratamiento de pacientes suicidas provoca en los profesionales sanitarios involucrados intensas reacciones emocionales, así como preocupaciones éticas y legales, que con frecuencia acontecen de forma inconsciente, aunque por ello no dejen de afectar su salud. A menos que estas reacciones puedan ser comprendidas, evaluadas y elaboradas adecuadamente, pueden sabotear el curso del tratamiento.

En 2003 la Fundación Americana para la Prevención del Suicidio, publicó una serie de recomendaciones para hacer frente a los problemas que produce la depresión en los estudiantes médicos, residentes y médicos, con una declaración de consenso y la facilitación de una documentación educativa (Struggling in Silence: Physician Depression and Suicide), sobre la necesidad de afrontar de forma eficaz la depresión en este colectivo profesional (Center, Davis, Detre et al. 2003). Este material está disponible en la Web de la fundación (www.afsp.org) y en www.doctorswithdepression.org, para facilitar la difusión de este material como herramienta educativa muy útil (Reynolds, Clayton 2009).

Hawton, Malmberg y Simkin (2004) proponen emplear un amplio rango de estrategias, que incluyen la mejora de la detección y tratamiento de los trastornos mentales, las medidas para reducir el estrés laboral y la restricción del acceso a medios de suicidio cuando los médicos están deprimidos. Desde un enfoque personalizado e interactivo, se realiza a través de la entrevista clínica con el paciente y sus familiares, a los que con frecuencia ha comunicado sus ideas de suicidio. El principal objetivo de estas entrevistas clínicas es el de llegar a hacer el diagnóstico del trastorno psiquiátrico existente 
en la mayoría de los casos con riesgo suicida. La impresión clínica de riesgo debe mediar en la decisión terapéutica de hospitalizar al paciente. Es importante también valorar la ocurrencia de sucesos vitales estresantes recientes graves, en relación con problemas interpersonales, familiares o laborales, así como la posible contribución del paciente a sus dificultades actuales y el papel potencial de la ayuda que se le puede proporcionar. Se estudiará también el estilo habitual del afrontamiento que utiliza el paciente para intentar resolver sus dificultades mayores.

Según la Guía de la OMS: "Preguntar sobre ideación/intención autolítica no provoca la conducta autolítica. A menudo reduce la ansiedad asociada con pensamientos o comportamientos autolíticos y ayuda al individuo a sentirse comprendido. Sin embargo, es preciso establecer una relación de confianza y de seguridad con la persona antes de preguntar y pedirle que trate de explicar con sus propias palabras sus motivos para querer hacerse daño" (OMS, Guía mhGAP).

En cuanto a los Antecedentes Personales se destacará la existencia de enfermedad mental, sobre todo depresión, alcoholismo, trastornos de personalidad, esquizofrenia; intentos de suicidio previos, es el factor de riesgo más importante, y los de abuso y maltrato en la infancia y adolescencia.

Se deben tener en cuenta también otros factores de riesgo: Enfermedades orgánicas, sobre todo si cursan con dolor crónico o son debilitantes o terminales.

Viudo, soltero o separado.

Vivir solo. Aislamiento social.

Falta de apoyos, especialmente no tener una persona de confianza.

Duelo o pérdida personal reciente o pérdida afectiva importante en la infancia.

Problemas familiares.

Desempleo, retiro o jubilación.

Problemas económicos/legales.

Acceso a medios.

Antecedentes familiares de suicidio o trastornos psiquiátricos.

Alta hospitalaria psiquiátrica reciente.

Desesperanza.

Para la valoración del riesgo autolítico Hawton y Catalan (1987) han descrito un esquema de entrevista semiestructurada que incluye la valoración de los diversos factores que ayudan a determinar el grado de riesgo de subsiguiente suicidio, la presencia de trastorno psicológico, y la naturaleza de los problemas actuales del paciente. En el modelo desarrollado en Oxford por Hawton y Catalan se emplea un enfoque orientado al problema con preferencia al puramente diagnóstico. El paciente asume un papel activo en definir sus problemas junto con el profesional que le valora, para ayudarle a comprender sus dificultades actuales y a desarrollar estrategias de afrontamiento adaptativas. Tras un intento de suicidio se tratarán de comprender las razones del mismo y las consecuencias buscadas, obteniendo una detallada exposición de los hechos que han precedido y seguido al intento. Para ello se recomienda:

1. Realizar un informe clínico completo tras explicar al paciente la finalidad de la entrevista.

2. Comprender el intento:

- Informe detallado de los hechos en las 48 horas previas al intento.

- Circunstancias que rodearon el acto: grado de planificación, aislamiento, nota suicida, motivos, acciones después del intento, ingesta de alcohol.

- Cociente riesgo/probabilidad de ser rescatado

- Intentos previos. 
3. Aclaración de las dificultades actuales:

- Naturaleza de los problemas, su duración, y recientes cambios.

- Facetas a cubrir: problemas psicológicos y físicos, relación con su pareja y con otros miembros de la familia, hijos, trabajo, amigos y consumo de alcohol.

4. Antecedentes:

- Historia familiar y personal relevante.

- Personalidad habitual.

5. Recursos disponibles:

- Recursos actuales para enfrentarse con su problema, recursos personales y recursos externos (tales como amigos, agencias sociales y médico de cabecera).

- Formas previas de hacer frente a las dificultades.

6. Valoración del estado mental en la entrevista:

- En especial estado de ánimo, ideación e intención suicidas, desesperanza y estado cognitivo.

7. Lista de problemas actuales: formularlos teniendo en cuenta las percepciones del paciente. Identificar posibles desencadenantes.

8. Establecimiento de más ayudas en caso necesario:

- Qué desea el paciente y qué está preparado a aceptar.

- Quién más debe estar implicado (por ejemplo, la pareja u otros parientes).

9. Pacto de no autoagresión y establecimiento del compromiso terapéutico. También se explicitarán los términos acordados en los que se impliquen otras personas o agencias.

Aunque es muy difícil prevenir algunos tipos de suicidio, todos los profesionales sanitarios debemos prestar la mayor atención a este grave problema de Salud Pública y aprender a tomar las medidas de seguridad más adecuadas a cada caso individual, y sobre todo en los siguientes casos: pacientes deprimidos con antecedentes personales y/o familiares de conducta suicida, si existe comorbilidad médica y psiquiátrica asociada o adicciones. Desde el punto de vista organizativo, es preciso que las instalaciones reúnan las condiciones más adecuadas para garantizar una contención eficaz y el control de los riesgos previsibles de forma individualizada. Para ello puede ser útil entender el comportamiento suicida desde un punto de vista dimensional. En estudios de población general se encuentra que un $20 \%$ de personas reconocen haber tenido al menos un episodio de ideación suicida moderada o severa, definida como ideas persistentes de suicidio que duran al menos 2 semanas, haciendo algún plan e identificando los medios necesarios. En población general se ha encontrado que $10 \%$ a $12 \%$ de los sujetos examinados admiten haber hecho al menos un intento previo de suicidio. Una gran mayoría de personas que hacen un intento de suicidio no acabarán consumándolo, con una clara falta de continuidad de una forma a otra de conducta suicida, aunque la repetición de los intentos se asocia con un riesgo mayor de suicidio consumado. Tan importante como la exposición a situaciones vitales estresantes, es tener dificultades para resolver problemas cotidianos por déficits de habilidades de afrontamiento, como para la solución de conflictos interpersonales, y el predominio de un estilo de afrontamiento pasivo-evitativo, en vez de los orientados a la identificación y solución proactiva de los problemas (Williams y Pollock 2000).

Según los datos del estudio OMS/EURO, la conducta suicida no fatal es bastante más frecuente que el suicidio consumado, sobre todo en encuestas anónimas realizadas a adolescentes, con una prevalencia vida del 3\% para las mujeres y el $2 \%$ para los varones, con algunas variaciones entre países y regiones, lo que apoya la hipótesis de que las conductas suicidas son diferentes métodos de intentar resolver problemas difíciles cuando no se cree disponer de recursos efectivos para resolverlos, aunque estos resultados son cuestionables por razones metodológicas (Schmidtke et al. 1996). 
El problema básico del clínico es valorar si un paciente realizará o no un intento de suicidio a corto plazo, habitualmente en las próximas 24-48 horas. La cuestión es como diferenciar los pacientes que piensan en el suicidio de aquellos otros que lo actuarán, lo que es bastante más infrecuente. Es fundamental evaluar en profundidad los indicadores de seria intención suicida que se enumeran a continuación:

Acto llevado a cabo sin que hubiera nadie.

En un momento en que era improbable que acudiera alguien.

Se tomaron precauciones para evitar ser descubierto.

Se hicieron preparaciones anticipando la muerte (testamento, seguros...).

Se preparó el acto (acceso a medios).

Se comunicó la intención a alguien con antelación.

Premeditación.

Se dejó una nota.

No se pidió ayuda después del acto.

Admisión de la intención suicida.

La entrevista clínica con la adecuada evaluación del riesgo de suicidio, el tratamiento eficaz y las medidas de seguridad apropiadas al paciente (manejo del paciente suicida individual) son los principales recursos para la prevención del suicidio.

Se han diseñado varios instrumentos que proporcionan información clínica útil de factores predictivos o de riesgo de suicidio, pero que no permiten definir un riesgo inminente de suicidio, sino solo identificar a un paciente como perteneciente al grupo de riesgo de suicidio. Escalas para la valoración psicométrica de los comportamientos suicidas:

- Escalas no específicas de suicidio

- MINI Entrevista Neuropsiquiátrica Internacional (MINI)

- Escala de Hamilton para la Depresión (HDRS)

- Escala de Depresión de Montgomery-Asberg (MADRS)

- Escalas específicas de evaluación del comportamiento suicida

- Escala de Ideación Suicida de Beck (SSI)

- Escala de Ideación Suicida de Paykel (PSS)

- International Suicide Prevention Trial Scale for Suicidal Thinking (InterSePT)

- Escala SAD PERSONS

- Escala Columbia para Evaluar el Riesgo de Suicidio (C-SSRS)

- Escala de Riesgo Suicida de Plutchik (RS)

- Escala de Impresión Clínica Global para Gravedad de Riesgo Suicida (CGI-SS)

- Escalas de Evaluación de Actos Suicidas

- Escala de Intencionalidad Suicida (SIS)

- Gravedad Médica de la Tentativa (MDS)

- Escalas para la evaluación de los constructos relacionados con el suicidio

- Escala de desesperanza (HS)

- Inventario de Razones para Vivir (RFL)

- Escala de impulsividad de Barrat (BIS-11)

- Inventario de Hostilidad de Buss- Durkee (BDHI)

- Historia de Agresión de Brown-Goodwin (BGLHA)

- Escala de Acontecimientos Vitales Estresantes de Brugha (LTE) 


\section{DUELO POR SUICIDIO}

El suicidio de un paciente produce un importante impacto emocional en sus médicos, con predominio de sentimientos de fracaso, insuficiencia y culpa, con frecuentes rumiaciones angustiosas sobre el hecho de no haberse dado cuenta durante la consulta de que el paciente puede haber tenido ideas de suicidio. El suicidio de un paciente constituye un importante acontecimiento vital estresante para el equipo asistencial y sus miembros deben afrontar el impacto de la pérdida así como las vivencias de incapacidad y fracaso por no haber podido evitarlo. Se trata de una probabilidad estadística cierta para todo el personal sanitario, pues un porcentaje significativo de las personas que fallecen por suicidio (en torno a un 40\%) han buscado atención médica en los 6 meses previos. El tratamiento de pacientes suicidas provoca intensas reacciones emocionales en los miembros del equipo asistencial, así como preocupaciones éticas y legales, que con frecuencia acontecen de forma inconsciente. A menos que estas reacciones puedan ser comprendidas, evaluadas y elaboradas, pueden sabotear el curso del tratamiento. Al contrario, contar con conocimientos y habilidades para la detección y el tratamiento de pacientes con riesgo de suicidio hace posible ayudarles, e incluso, si se trata del propio médico o de otro compañero, buscar la ayuda profesional más adecuada.

La comunicación del suicidio desencadena en todos los miembros del equipo terapéutico un complejo proceso de reacciones vivenciales que ha sido revisado por Padierna Acero (1990). Transcurre de forma habitual en varios estadios, en primer lugar, la reacción inmediata de "shock" o de impacto agudo con sentimientos de incredulidad, negación, despersonalización y aislamiento emocional, o bien de angustia y sentimientos de culpa. Después tienen lugar diferentes reacciones de duelo tanto en el ámbito personal como profesional, que van a depender de múltiples factores, como son la calidad de la relación previa con el paciente, variables de personalidad y experiencia profesional individual, así como de la calidad de las relaciones interpersonales dentro del equipo o soporte social profesional.

El duelo personal acontece como un proceso privado aunque trascienda en la relación con los demás compañeros y con los propios familiares. El duelo profesional puede facilitarse a través de ciertas prácticas institucionales como son las sesiones clínicas de revisión del caso y la consulta con un supervisor, o con otros compañeros que facilitan la elaboración del trabajo de duelo personal, mejorar la comunicación, competencia y soporte del equipo, y reevaluar y mejorar la calidad asistencial y del trabajo de prevención del suicidio de pacientes con riesgo autolítico. El proceso de elaboración de un duelo normal incluye sentimientos de ansiedad y tristeza, pero la autoestima permanece indemne, sin sentimientos generales de autodesvalorización, deterioro de las relaciones interpersonales ni de los estándares asistenciales. El duelo normal es un proceso autolimitado que media en el logro de una adecuada recuperación personal y reorganización funcional, con posibilidad de aprender de la experiencia y de mejorar la práctica asistencial.

\section{CONCLUSIONES}

En nuestro país no existe un registro fiable de la distribución de las muertes por suicidio según la profesión, debido a que no se cumplimenta el certificado de defunción con el mismo rigor que se hace en el caso de accidentes de tráfico y homicidios. Es necesario que la Organización Médica Colegial (OMC) inste a los médicos a hacerlo adecuadamente, para conocer la influencia de los riesgos psicosociales del trabajo en la salud del empleado y poder tomar medidas preventivas eficaces. Se recomienda que la OMC proponga al Ministerio de Sanidad y al Instituto Nacional de Estadística que se exija el cumplimiento estricto del certificado de defunción, como en cuanto a la causa fundamental o etiológica y la ocupación o profesión de la persona que se haya suicidado. 
Es preciso documentar correctamente en la historia clínica la evaluación del estado mental y del riesgo de suicidio, en especial después de un intento.

Todas las campañas de prevención del suicidio destacan la relevancia de diagnosticar y tratar adecuadamente la depresión como medida preventiva fundamental y también de la importancia de luchar contra el estigma social asociado a la enfermedad mental.

La mayor tasa de suicidio de los médicos y especialmente en mujeres, hace necesario mejorar su formación en los aspectos psicosociales inherentes a la práctica clínica desde la formación de grado hasta la jubilación, promover el cuidado de la salud del médico para que pueda cuidar adecuadamente de la de los demás, así como mejorar sus condiciones de trabajo del para garantizar la seguridad del usuario tanto como la del profesional sanitario.

Es necesario garantizar la autonomía profesional de los médicos en la toma de decisiones clínicas según la evidencia científica, igual que la del resto de profesionales sanitarios, para lo que se deberán implementar medidas para la participación eficaz de los mismos en la planificación, regulación y control de la organización y gestión de los centros sanitarios. De ello depende no sólo la calidad asistencial y la seguridad de los enfermos, sino también la salud de los profesionales. Las condiciones y la organización del trabajo condicionan tanto la calidad asistencial como la calidad laboral de los trabajadores que les atienden. Igualmente es fundamental promover la formación continuada del médico, la supervisión eficaz, el trabajo en equipo y el desarrollo efectivo de la carrera profesional.

Siguiendo las recomendaciones de la Estrategia en Salud Mental del Sistema Nacional de Salud, para prevenir la enfermedad mental, las adicciones y el suicidio, se deben realizar y evaluar acciones específicas para disminuir las tasas de depresión, adicciones y suicidio en grupos de riesgo. Para ello, se deben desarrollar intervenciones preventivas, como talleres específicos y entrenamiento en habilidades para la prevención y tratamiento de estos graves problemas de salud pública.

\section{BIBLIOGRAFÍA}

Aasland OG, Ekeberg O, Schweder T. Suicide rates from 1960 to 1989 in Norwegian physicians compared with other educational groups. Social Science Medicine 52 (2): 259-265, 2001.

Agerbo E, Gunnell D, Bonde JP et al. Suicide and occupation: the impact of socio-economic, demographic and Psychiatric differences. Psychological Medicine 37: 1131-1140, 2007.

Aguilar Shea AL, Vera García M, Outumuro Cadavid A et al. La salud de los médicos de familia: ¿practicamos lo que predicamos? Atención Primaria 43 (6):305-311, 2011.

American Academy of Family Physicians. Lifestyle/Personal Health Care in Diferent Occupations. Kansas City, Mo, 1979.

American Medical Association. Council on Mental Health. The sick physician. Impairment by psychiatric disorders. JAMA 223: 684687, 1973.

American Medical Association. Council on Scientific Affairs. Physician mortality and suicide: results and implicatios of the AMA-APA pilot study. Conn Med 50:37-43, 1986.

Arendt H. Sears in Understanding. Ed. Harcourt Brace. New York, 1994.

Arensman E, Kerkhof A, Hengeveld M. et al. Medically treated suicide attempts: a four year monitoring study of the epidemiology in the Netherlands. Journal of Epidemiology and Community Health, 49: 285-289, 1995.

Arnetz BB, Horte LG, Hedberg A, et al. Suicide patterns among physicians related to other academics as well as to the general population. Results from a national long-term prospective study and a retrospective study. Acta Psychiatrica Scandinavica 75 (2): 139-143, 1987.

Bandura A. Teoría del Aprendizaje Social. Ed. Espasa Calpe, Madrid, 1987.

Barcia D. Necesidad de una medicina antropológica. Publicaciones Universidad de Murcia, 1979.

Barraclough B, Bunch J, Nelson B, Sainsbury P. British Journal Psychiatry 112. 355, 1974. Citado en rucinski y Cybulska, 1985. 
Beck AT, Steer RA, Kovacs M, Garrison B. Hopelessness and eventual suicide: a 10-year prospective study of patients hospitalized with suicidal ideation. American Journal Psychiatry 142 (5): 559-563, 1985.

Blach CM, Oreskovich MR, Dyrbye LN et al. Personal consequences of malpractice lawsuits on American surgeons. Journal College Surgeons 213(5): 657-667, 2011.

Blazer DG, Kessler RC, McGonagle KA et al. The prevalence and distribution of major depression in a national community sample: the National comorbidity Survey. American Journal Psychiatry 151: 979-986, 1994.

Blumenthal D, Gokhale M, Campbell E, Wissman J. Preparedness for clinical practice. JAMA: 1027-1034, 2006.

Bovier PA, Arigoni F, Schneider M, Gallachi MB. Relatioships between work satisfaction, emotional exhaustation and mental Health among Swiss primary care physicians. European Journal Public Health 19 (6): 611-617, 2009.

Bobes García J, Sáiz Martínez PA, García-Portilla MP, Bascarán Fernández MT, Bousoño García M. Comportamientos suicidas. Prevención y tratamiento. Ars Medica, Barcelona, 2004

Bousoño García M, García prieto A, Bascarán Fernández MT. Aspectos bioéticos y morales, en: Julio Bobes García, Pilar Alejandra Sáiz Martínez, María Paz García-Portilla González, María Teresa Bascarán Fernández, Manuel Bousoño García. Comportamientos suicidas. Prevención y Tratamiento. Ed. Ars Médica. Barcelona, 2004.

Boyd JH \& Weissman MM. The epidemiology of affective disorders: A reexamination and future directions. Archives of General Psychiatry 38, 1039-1046, 1981.

Center C, Davis M, Detre T, et al. Confronting depression and suicide in physicians: a consensus statement. JAMA 289: 3161-3166, 2003.

Chan AO, Huak CY. Influence of work environment on emotional health in a health care settings. Occupational Medicine 54 (3):207-212, 2004.

Charlton J, Kelly S, Dunnell K et al. Suicide deaths in England and Wales: trends in factors associated with suicide deaths. Populations Trends 71: 34-42,1993.

Chiles JA, Strosahl Kd. The suicidal patient. American Psychiatric Press. Inc. Washington, 1995.

Cornette MM, deroon-Cassini TA, Fosco GM, et al. Application of an interpersonal-psychological model of suicidal behavior to physicians and medical traines. Archives Suicide Research 13 (1): 1-14, 2009.

Croasdale M. Women foun more likely to burn out from practice stress. American Medical News 48.1-2, 2005.

de la Cruz C, Corominas A, Sarró B. El suicidio en la profesión médica: revisión bibliográfica. Monografías Médicas Jano, 2 ( $\mathrm{n}^{\circ}$ 9), 1988.

De la Monte SM, Hutchins GM. Is suicide a special occupational hazard for physicians?

JAMA 251 (15):1952-3.

Dublin LI, Spiegelman M. The longevity and mortality of American physicians. JAMA 9: 1211-1215, 1947.

Ducker DG. Role conflict for women for women physicians. En CD Scott and JE Hawks (eds), Heal Thyself: The Health of two sources of the Health Professional, Brunner-Mazel, New York. 1987.

Epstein, RM, Hundert, EM. Defining ans assessing professional competente. JAMA 2002, Jan 9; 287 (2): $226-235$.

Epstein, RM et al. Comprenhensive assessemante of professional competente: the Rochester experiement. Teach learn med. 16 (2): 186-196, 2004.

Firth-Cozens J. Emotional distress in junior house officers. British Medical Journal 295: 533-536, 1987.

Ford De, Mead LA, Chang PP et al. Depression is a risk factor for coeonary artery disease in men: the precursors study. Archives Internal Medicine 158: 1422-1426, 1998.

Frank E, Dingle AD. Self-reported depression and suicide attemts among US women physicians. American Journal Psychiatry 156: 1887-1894, 1999.

Fridner A, Belkic K, Marini M. et al. Survey on recent suicidal ideation among female university hospital physicians in Sweden and Italy (the HOUPE study): cross-sectional associations with work stressors. Gender Medicine 6 (1): 314-328, 2009.

Fridner A, Belkic K, Minucci D et al . Work Environment and recent Suicidal Thoughts Among Male University Hospital physicians in Sweden and Italy: The health and organization among University Hospital physician in Europe (HOUPE) Study. Gender Medicine 8 (4): 269-279, 2011.

Gabbard GO. The role of Compulsiveness en the Normal Physician. JAMA 254: 2926-2929, 1985.

Gabbard GO. Psiquiatría Psicodinámica en la Práctica Clínica. Ed. Médica Panamericana. Buenos Aires, 2002.

Gabbard GO, Allison SE. Psychodinamic treatment, en American Psychiatric Publishing Textbook of Suicide. Assessment and Management. Ed. Simon RI, Hales RE. Washington, DC, American Psychiatric Publishing, 2006.

Gagné P, Moamai J, Bourget D. Psychopatology and Suicide among Quebec Physicians: A Nested Case Control Study. Depression Research and Treatment. 2011. 
Gist ME, Mitchell TR. Self-efficacy: a theoretical analysis of its determinants and malleability. Academy of Management Review 17(2): 183-211, 1992.

Gold KJ, Sen A, Schwenk TL. Details on suicide among US physicians: data from the National Violent Death Reporting System. General Hospital Psychiatry 35:45-49, 2013.

Gracia D. Fundamentos de bioética. Ed. Eudema, Madrid, 2007.

Gyorffy Z, Adám S, Csoboth C, Kopp M. The prevalence of suicide ideas and their psychological backgrounds among physicians. Psychiatrie Hungarian 20 (5): 370-379, 2005.

Hassan TM, Ahmed SO, White AC, Galbraih N: A postal survey of doctors' attitudes to becoming mentally ill Clinical Medicine 9 (4): 327-332, 2009.

Hawton K. and Catalan J. Attempted suicide: a practical guide to its nature and management ( $2^{\text {nd }}$ edn). Oxford University Press, 1987.

Hawton K, Clements A, Sakarovitch C et al. Suicide in doctors: a study of risk according to gender, seniority and speciality in medical practitioners in England and Wales 1979-1995. Journal Epidemiology Community Health 55:296-300, 2001

Hawton K, van Heeringen K. Suicide. The Lancet 373: 1372-1381, 2009

Heikkinen M, Aro H, Lonnqvist J. Recent life events, social support and suicide. Acta Psychiatrica Scandinavica Suppl. 377: 65-72, 1994

Heim E. Job stressors and Coping in Health Professions. Psychotherapy and psychosomatics 55: 90-99, 1991.

Hem E, Gronvold NT, Aasland OG et al. The prevalence of suicidal ideation and suicidal attempts among Norwegian physicians. Results from a cross-sectional survey of a national sample. European Psychiatry 15 (3): 183-189, 2000

Hem E, Haldorsen T, Aasland OG et al Suicide rates according to education with a particular focus on physicians in Norway 1960-2000 psychological medicine 35 (6): 873-880, 2005.

Hernández M, Ayuso Mateos JL. Programa de Formación profesionales Sanitarios de la CAM que trabajan con pacientes en edad geriátrica sobre detwección y Prevención de la Depresión y el Suicidio. Ministerio de Sanidad, Madrid, 2010.

Joiner T, Brown JS, Wingate LR. The psychology and Neurobiology of suicidal behavior. Annual Review Psychology 56: 287-314, 2005.

Karasek et al. Jobs demands, job decision latitude and mental strain: Implications for job desing. Administrative Science Quarterly 24. 285-308, 1979

Kauppinen-Toropainen K. et al. Job dissatisfaction and work-related exhaustion in male and female work. Journal of Occupational Behavior 4: 193-207, 1983

Kelly S and Bunting J. Trends in suicide in England and Wales, 1982-96. Populatios Trends 92: 29-41, 1998.

Kessler RC, Berglund P, Demler O, Jin R, Merikangas KR, Walters EE. 2005. Lifetime prevalence and age-ofonset distributions of DSM-IV disordes in the National Comorbidity Survey Replication. Arch. Gen. Psychiatry 62(6):593-602.

King H. Health in the medical and other learned professions. Journal chronic Disease 23: 257-281, 1970.

Kotler M, Iancu i, Efroni R et al. Anger, impulsivity, social support, and suicide risk in patients with posttraumatic stress disorder. Journal Nervous Mental Disorder 189: 162-167, 2001.

Lagro-Janssen AL, Luijs HD. Suicide in female and male physicians. Ned Tijdschr Geneeskd. 152 (40): $2177-2181,2008$.

Lindeman S, Laara E, Hakko H et al 1996. A sistematic review on Gender-specific suicide mortality in medical doctors. British Journal Psychiatry 168: 274-279, 1996.

Lindeman S, Heinanen H, Vaisanen E et al. Suicide among medical doctors: Psychological aurtopsy on seven cases. Archives Suicide Research 4: 135-141, 1998.

Lindfors PM, Meretoja OA, Luukkonen RA et al. Suicidality among Finnish anaesthesiologists. Acta Anaesthesioloigy Scandinavian 53: 1027-1035, 2009.

López Sánchez F, Carpintero Raimundez E, del Campo Sánchez A, Lázaro Visa S, Soriano Rubio S. El bienestar personal y social y la prevención del malestar y la violencia. Ed. Pirámide. Madrid, 2006.

Maltsberger JT. Outpatient treatment en The American Psychiatric Publihing Textbook of Suicide Assessment and Management. Ed. Simon RI, Hales RE. Washington, DC, American Publishing, 2006.

Mann JJ, Oquendo MA, Underwood MD, Arango V. The neurobiology of suicide risk: A review for the clinician. Journal Clinical Psychiatry 60: 7-11,1999.

Mann JJ, Waternaux C, Haas GL, et al. Toward a clinical model of suicidal behavior in Psychiatric patients. American Journal Psychiatry 156: 181-189, 1999. 
Marina JA. Dictamen sobre Dios. Ed. Anagrama. Barcelona, 2001.

Marina JA, de la Válgoma M. La magia de leer. Ed. Plaza Janés, Random House Mondadori, SA. Barcelona, 2005.

May HJ, Revicki DA. Professional Stress Among Family Physicians. The Journal of Family Practice 20: 165-171, 1985.

Mc Murray JE, Linzer M, Konrad TR, et al. The work lives of women physician: a results from the physician work life Study. The SGIM Career Satsfaction Study Group. Journal General Internal Medicine 15: 372-380, 2000.

Mingote Adán JC. Satisfacción, estrés laboral y calidad de vida del médico. Tesis Doctoral, Universidad Autónoma de Madrid, 1995.

Mingote Adán JC, Jiménez Arriero MA, Osorio Suárez R, Palomo T. Suicidio. Asistencia clínica. Guía práctica de psiquiatría médica. Ed. Diaz de Santos, Madrid, 2004.

Mingote C, Requena M (Editores). El malestar de los jóvenes. Contextos, Raíces y Experiencias. Ed. Díaz de Santos, Madrid, 2008.

Mingote Adán JC, del Pino Cuadrado P, Gálvez Herrer M y cols. Utilidad preventiva del constructo "trastorno mental grave" en el ámbito sociosanitario. Medicina y Seguridad del Trabajo 56 (221): 306-322, 2010.

Müller N, Myint AM, Schwarz MJ. Inflammatory Biomarkers and Depression, en: Tomás Palomo, Richard M Kostrzewa, Richard J Beninger (Eds.). Staging Neuropsychiatric Disorders. Implications for Etiopathogenesis and Treatment. Springer. Madrid, 2012.

Myers MF. Physician suicides leave many victims in their wake. Winnipeg Free Press, October 1, 2006.

Myers MF, Gabbard GO. The Physician as Patient. A Clinical Handbook for Mental Health Professionals. Ed. American Psychiatric Publishing, Inc. Washington, 2008.

Neighbour R. La Consulta Interior. Ed. J\&C S.L. Esplugues de Llobregat, 1998.

Nogales, A., García-Seoane, J., Calvo, E. y Grupo de Trabajo Para la Definición de Competencias. Competencias para el Grado de Medicina de la Universidad Complutense de Madrid. Documento Base. Unión Editorial: Madrid 2008.

New Zealand Guidelines Group and Ministry of Health. The assessment and management of people at risk of suicide, Wellington: New Zealand Guidelines Group and Ministry of Health. 2003.

Nierenberg AA, Gray SM, Grandin LD. Mood disorders and suicide. Journal Clinical Psychiatry 62 (Suppl 25): 27-30, 2001.

Ortega y Gasset J. La rebelión de las masas. Ed. Austral, Madrid, 1929.

Owen D. Diseased, demented, depressed: serious illness in Heads of State. Ocupacional Medicine 53: $491-501,2003$.

Petersen MR, Burnett CA. The suicide mortality of working physicians and dentists. Occupational Medicine (Lond.) 58 (1): 25-29, 2008.

Pompili M, Innamorati M, Narciso V. et al. burnout, hopelessness and suicide Risk in medical doctors. Clínica Terapeutica 161 (6): 511-514, 2010.

Post RM. Mechanisms of Illness Progression in the Recurrent Affective Disorders, en: Tomás Palomo, Richard M Kostrzewa, Richard J Beninger (Eds.). Staging Neuropsychiatric Disorders. Implications for Etiopathogenesis and Treatment. Springer. Madrid, 2012.

Preven DW. Physician Suicide. The Psychiatrist's Role, en The Impaired Physician (ed) Stephen C Scheiber and Brian B Doyle Plenum Medical Book Co., New York, 1983.

Registrar General. Decenial Supplement, England and Gales 1959-63 on Occupational Mortality, HMOS, London, 1978.

Retorta J. El poder y sus conflictos. Ed. Plural Paidós. Barcelona, 2005.

Sadock, B.J. y Sadock, V.A. Sinopsis de Psiquiatría. Waverly Hispánica S.A. Barcelona, 2004.

Revicki DA, May HJ. Development and Validation of the Physician Stress Inventory Family Practice Research Journal 2:211-225, 1983.

Revicki DA, May HJ. Occupational stress, Social Support, and Depression. Health Psychology 4: 61-77, 1985.

Revicki DA, May HJ, Whitley TW. Reliability and Validity of the work-Related Strain Inventory Among Health Professionals. Behavioral Medicine 12: 111-120,1991.

Reynolds Ch, Clayton P. Out of the Silence: Confronting Depression in Medical Students and Residents. Academic Medicine 84 (2): 159-160, 2009.

Rich CL, Pitts FN. Suicide by male physicians during a five-year period. American Journal psychiatry 136.1089-1090, 1979.

Rich CL, Pitts FN. Suicide by psychiatrist: A study of medical specialists among 18,730 consecutive physician deaths during a 5 year period (1967-1972). Journal Clinical Psychiatry 41: 261-263, 1980. 
Richards C. The health of doctors. King Edward`s Hospital Fund for London, 1989.

Rose KD and Rosow I. Physicians who kill themselves. Archives General Psychiatry 29: 800, 1973.

Ritsher JB, Otilingam PG, Grajales M. Internalized stigma of mental illness: psychometric properties of a new measure. 121: 31-49, 2003.

Ross M. Suicide and the psychiatrist. American Journal Psychiatry 130: 937, 1973.

Roskar S, Podlesek A, Zorko M, et al. Effects of training on recognition and management of drpression and suicide risk evaluation for Slovenian primary-care physicians: follow-up study. Croatian Medical Journal 51 (3): 237-242, 2010.

Rucinski J and Cybulska E. Mentally ill doctors British Journal Hospital Medicine, II, 1985.

Sarró B y de la Cruz C. Los suicidios. Martínez Roca, Barcelona, 1991.

Shanafelt TD, Balch CM, Dyrbye L, et al. Special report: suicidal ideation among American surgeons. Archives Surgery 146 (1): 54-62. 2011.

Satcher D. From the Surgeon General. JAMA, 284: 950, 2000

Schmidtke A, Bille Brahe U, De Leo D et al. Attempted suicide in Europe: rates, trends and sociodemographic characteristics of suicide attempters during the period 1989-1992. Results of the WHO/EURO Multicentre Study on Parasuicide: Acta Psychiatrica Scandinavica 93, 327-338, 1996.

Schernhammer ES, Colditz GA: Suicide rates among physicians: a quantitative and gender assessment (metaanalysis). American Journal Psychiatry 161: 2295-2302, 2004.

Siegrist J, Rödel A. Work stress and health risk behavior. Scandinavian Journal Work Environmental Health 32 (6): 473-481, 2006.

Silverman M. Physicians and suicide, en The Handbook of Physician Health: An Essential Guide to Understanding the Healthcare Needs of Physicians. Ed. Goldman LS, Myers M, Dickstein LJ. Chicago, IL, American Medical Association, 2000.

Simon RI. Patient safety versus freedom of movement: coping with uncertainty, en The American Psychiatric Publihing Textbook of Suicide Assessment and Management. Ed. Simon RI, Hales RE. Washington, DC, American Publishing, 2006.

Spector PE. Behavior in Organizations as a function of employee`s locus of control. Psychological Bulletin 91 (3):482-497, 1982.

Steppacher RC and Mausner JS. JAMA 228. 323,1974. Citado en Rucinski y Cybulska (1985).

Torre D, Wang NY, Mead LA, et al. Mortality in a prospective study of physicians. Journal General International Medicine 15 (suppl): 150, 2000.

Tyssen R, Hem E, Vaglum P, et al. The process of suicidal planning among medical doctors: predictors in a longitudinal Norwegian sample. Journal Affective Disoeders 80 (2-3): 191-198, 2004.

Vaillant GE. Adaptation to Life. Little Brown and Co. Inc. Boston, 1977.

Vaillant GE, Vaillant CO. Natural History of Male psychological health XII: A 45 Year Study of Predictors of Successful Aging at Age 65. American Journal of Psychiatry 147: 31-37, 1991.

Van der Heijden F, Dillingh G, Bakker A, et al. Suicidal Thoughts Among Medical Residents with Burnout. Archives of Suicide Research 12: 344-346, 2008.

Wada K, Yoshikawa T, Goto T et al. Association of Depression and Suicidal Ideation with Unreasonable Patient Demands and Complaints Among Japanese Physicians: A National Cross-sectional survey International Journal Behavioral medicine 18: 384-390, 2011.

Williams JMG and Pollock LR. The psychology of suicidal behavior, en The internal handbook of suicide (ed. K Hawton and C Van Heeringen). Wiley, Chichester, 2000.

Wortman C, Biernat M, Lang P. Coping with Role Overload en Frankerhaeuser M, Lundberg E, Chesney M. Women, Work and Health. Stress and Opportunities. Plenum Press, New York 1991. 